\title{
Approximation-Based Robust Adaptive Backstepping Prescribed Performance Control for a Huger Class of Nonlinear Systems
}

\author{
Jihui Xu, Xiaolin Wang $(\mathbb{D}$, and Lei Zhang \\ Equipment Management and UAV Engineering College, Air Force Engineering University, Xian 710051, China \\ Correspondence should be addressed to Xiaolin Wang; wxl0821@outlook.com
}

Received 1 March 2019; Revised 24 May 2019; Accepted 25 June 2019; Published 25 August 2019

Academic Editor: Sergey Dashkovskiy

Copyright ( 2019 Jihui Xu et al. This is an open access article distributed under the Creative Commons Attribution License, which permits unrestricted use, distribution, and reproduction in any medium, provided the original work is properly cited.

\begin{abstract}
This paper proposes an innovative adaptive neural prescribed performance control (PPC) scheme for large classes of nonlinear, nonstrict-feedback systems under input saturation constraint. A restrictive hypothesis under which the upper and lower bounds of control gain functions exist a priori is first relieved by constructing appropriate compact sets within which all state trajectories are held. A novel asymmetry error transformed variable is then introduced to cope with the nondifferentiable obstacle and complex deductions corresponding to traditional PPC schemes. To efficiently manage the input saturation constraint, a new auxiliary dynamic system with a bounded compensation tangent function term is established as the strictly bounded assumption of the dynamic system is canceled. It is rigorously proven that all signals in the closed-loop systems are semiglobally uniformly ultimately bounded under both Lyapunov and invariant set theories. The tracking errors converge to a small tunable residual set with prescribed performance under the effect of the input saturation constraint. The effectiveness of the proposed control scheme is thoroughly verified by two simulation examples.
\end{abstract}

\section{Introduction}

The approximation-based adaptive control of uncertain nonlinear systems is a significant theoretical challenge that has garnered a great deal of research interest in recent years [13]. Many researchers have utilized fuzzy logic systems or neural networks (NNs) for this purpose [4-10]. When combined with the backstepping methodology, approximationbased adaptive approaches can achieve global stability for many classes of nonlinear systems [11-15]. However, as the traditional backstepping controller repeatedly differentiates the virtual controllers at each step, its complexity drastically increases as the order of systems increases. The dynamic surface control (DSC) technique is designed to mitigate this problem by introducing a first-order low-pass filter at each step. The DSC technique has allowed scholars to construct approximation-based adaptive control schemes for many nonlinear strict-feedback and nonstrict-feedback systems [16-21]; however, these control schemes are all based on the assumption that the control gain functions must be bounded. To relax this restrictive hypothesis, two adaptive NNs control schemes were developed for strict-feedback and nonstrictfeedback systems by assuming that control gain functions are continuous and are bounded on a compact set [22, 23]. However, these schemes do not consider the simultaneous occurrence of prescribed performance and input saturation constraints due to inherent difficulties in the design.

The prescribed performance constraint is unavoidable in many industrial control systems, such as precise microinstruments and robotics, and may result in degradation, hazards, or system failure $[24,25]$. Thus, it represents an issue worth of careful attention during the control system design process [26]. The prescribed performance control (PPC) concept has been extensively employed in controller design for systems of various forms [27-35]. For example, in [27], an adaptive control scheme for SISO strict-feedback nonlinear systems capable of guaranteeing prescribed performance bounds is considered. In [30], an improved prescribed performance control scheme is proposed for a strict-feedback nonlinear dynamic system based on the backstepping technique. Recently, an adaptive NN-based decentralized control scheme under a prescribed performance constraint was recently presented 
for uncertain switched nonstrict-feedback interconnected nonlinear systems [35]. These control schemes share the common assumption that bounded control gain functions are required, which is very restrictive [27-35].

The input saturation constraint also appears in many actual systems, such as industrial robots and numerical control machines [36-38]. If it is ignored during control system design, it can cause inaccuracy, instability, or even deteriorate the performance of the entire closed-loop system. The input saturation problem has received much scholarly interest in recent years [39-43]. For example, an adaptive neural control method is proposed for a class of strictfeedback stochastic nonlinear system in the presence of input saturation constraint in [40]. Moreover, in [42], an adaptive fuzzy prescribed performance control scheme is presented for a class of nonstrict-feedback systems subject to input saturation constraint. Recently, an efficient fuzzy controller for a larger class of stochastic nonlinear systems is constructed to manage the input saturation constraint in [43]. However, previously published techniques [39-43] depend upon the assumption that the control gain functions are always bounded, which is overly restrictive; the upper and lower constants bounds of the control gain functions may be difficult to acquire in some practical systems or may even be nonexistent [41-43].

To the best of our knowledge, there are extremely few extant schemes applicable to the control of large classes of nonstrict-feedback systems under both prescribed performance and input saturation constraints where the control gain functions are possibly unbounded. This is yet an open problem with theoretical and practical significance. In this study, we developed a novel adaptive neural PPC scheme for a large class of nonlinear nonstrict-feedback systems with both prescribed performance and input saturation constraints. The main contributions of our work can be summarized as follows.

(1) Unlike other strategies [27-43], this is the first instance in which an adaptive control problem of a large class of nonstrict-feedback systems with continuous, possibly unbounded control gain functions fall under both prescribed performance and input saturation constraints.

(2) As discussed in this paper, we constructed a novel error constraint transformation to overcome the nondifferentiable obstacle and complex deductions existing in traditional PPC schemes. We also obtained a new asymmetry error constraint variable.

(3) By contrast to traditional backstepping techniques, no additional first-order filter or repeated differentiations of intermediate control signals are needed to operate the proposed technique. NNs are utilized to approximate the unknown continuous functions, which contain all states of the whole system.

The remainder of this paper is organized as follows. Section 2 provides the problem formulation and preliminaries. The adaptive neural prescribed performance controller is discussed in Section 3. Section 4 is devoted to stability analysis. In Section 5, two simulation examples are presented to demonstrate the effectiveness of the proposed control scheme. Section 6 concludes the paper.

\section{Problem Statement and Preliminaries}

2.1. Problem Formulation. Consider the following large class of uncertain nonstrict-feedback nonlinear systems [41]:

$$
\begin{aligned}
& \dot{\xi}_{j, i_{j}}=h_{j, i_{j}}\left(\bar{\xi}_{j, \rho_{j}}\right)+\theta_{j, i_{j}}\left(\bar{\xi}_{j, i_{j}}\right) \xi_{j, i_{j}+1}+D_{j, i_{j}}(\xi, t), 1 \leq i_{j} \leq \rho_{j}-1 \\
& \dot{\xi}_{j, \rho_{j}}=h_{j, \rho_{j}}\left(\bar{\xi}_{j, \rho_{j}}\right)+\theta_{j, \rho_{j}}\left(\bar{\xi}_{j, \rho_{j}}\right) u_{j}\left(\iota_{j}\right)+D_{j, \rho_{j}}(\xi, t) \\
& y_{j}=\xi_{j, 1}, \quad j=1,2, \ldots, m
\end{aligned}
$$

where $\xi_{j, i_{j}} \in R$ is the state of $j$ th subsystem and $\xi=$ $\left[\bar{\xi}_{1, \rho_{1}}^{T}, \ldots, \bar{\xi}_{j, \rho_{j}}^{T}, \ldots, \bar{\xi}_{m, \rho_{m}}^{T}\right]^{T} \in R^{N}$ represents the state vector of the whole system $\left(N=\rho_{1}+\cdots+\rho_{m}\right)$, where $\bar{\xi}_{j, \rho_{j}}^{T}=$ $\left[\xi_{j, 1}, \cdots, \xi_{j, \rho_{j}}\right]^{T} \in R^{\rho_{j}}$ and $\rho_{j}$ is the order of the $j$ th subsystem. $\bar{\xi}_{j, i_{j}}^{T}=\left[\xi_{j, 1}, \cdots, \xi_{j, i_{j}}\right]^{T} \in R^{i_{j}}, u_{j} \in R$, and $y_{j} \in R$ denote the system input and output of the $j$ th subsystem, respectively. $h_{j, i_{j}}(\cdot)$ are unknown continuous functions with $h_{j, i_{j}}(0)=0$; $\theta_{j, i_{j}}(\cdot)$ are unknown continuous control gain functions and $D_{j, i_{j}}(\xi, t)$ are uncertainties consisting of dynamical coupling terms and external disturbances. The system states are all assumed to be available in the control design process. Input saturation $u_{j}\left(\iota_{j}\right)$ can be written as follows:

$$
u_{j}\left(\iota_{j}\right)=\operatorname{sat}\left(\iota_{j}\right)= \begin{cases}\operatorname{sign}\left(\iota_{j}\right) u_{j, M}, & \iota_{j} \geq u_{j, M} \\ \iota_{j}, & \iota_{j}<u_{j, M}\end{cases}
$$

where $u_{j, M}$ means the bound of $u_{j}\left(\iota_{j}\right), \iota_{j} \in R$ is the input to the saturator, and $u_{j}=u_{j}\left(\iota_{j}\right)$.

Control Objective: the purpose of this study is to establish a novel adaptive neural prescribed performance controller $u_{j}$ for System (1) to guarantee two performance indicators. (1) All closed-loop signals in the systems are semiglobally uniformly ultimately bounded (SGUUB) and (2) the prescribed output tracking error bound for the tracking error, $e_{j, 1}(t)=y_{j}(t)-r_{j}(t)$, is always satisfied. In this paper, the given reference signal $r_{j}$ satisfies Assumption 1 .

Assumption 1. The reference signal $r_{j}$ is a sufficiently smooth function and there exists a constant $\mathfrak{R}_{j 0}>0$ such that $\prod_{j 0}:=$ $\left\{\left(r_{j}, \dot{r}_{j}, \ddot{r}_{j}\right)^{T} \mid r_{j}^{2}+\dot{r}_{j}^{2}+\ddot{r}_{j}^{2} \leq \mathfrak{R}_{j 0}\right\}$.

Definition 2 (see [18]). The solution of System (1) is SGUUB if, for any $\Pi$, a compact subset of $R^{n}$, and all $\bar{\xi}_{n}\left(k_{0}\right) \in \Pi$ there exist an $\zeta>0$ and a number $N\left(\zeta, \bar{\xi}_{n}\left(k_{0}\right)\right)$ such that $\left\|\bar{\xi}_{n}(k)\right\| \in \zeta$ for all $k \geq k_{0}+N$.

Unlike other methods [27-43], we aim here to achieve this objective in the presence of the following assumptions and lemmas.

Assumption 3. The continuous control gain functions satisfy $\theta_{j, i_{j}}\left(\bar{\xi}_{j, i_{j}}\right)>0$ for $i_{j}=1,2, \ldots, \rho_{j}, j=1,2, \ldots, m$. 
Remark 4. Unlike other scholars [27-43], we assume only the signs of the control gain functions to be known. This effectively relaxes the a priori boundedness assumption. Despite some other techniques available to relax this assumption $[22,23]$, the case has limited application in practice if the prescribed performance constraint and input saturation constraint are not taken into account. This complicates the control design of nonlinear systems due to the couplings between the output and input constraints.

Remark 5. To the best of our knowledge, the present study marks the first time that the adaptive control problem of a general class of nonstrict-feedback nonlinear systems with both prescribed performance and input saturation constraints has been investigated.

Assumption 6. For $\forall t>0$, there exist unknown constants $D_{j, i_{j}}^{*}>0$ satisfying $\left|D_{j, i_{j}}(\xi, t)\right| \leq D_{j, i_{j}}^{*}\left(i_{j}=1,2, \ldots, \rho_{j}, \quad j=\right.$ $1,2, \ldots, m)$.

Lemma 7 (see [12]). Consider the following dynamic system:

$$
\dot{\zeta}(t)=-a \varsigma(t)+p v(t)
$$

where $a$ and $p$ are positive constants and $v(t)$ is a positive function. For $\varsigma(0) \geq 0$, we have $\varsigma(t) \geq 0, \forall t \geq 0$.

Lemma 8 (see [15]). The hyperbolic tangent function $\tanh (\cdot)$ is uninterrupted and differentiable. For $\forall \chi \in R, \forall \varepsilon>0$, we have

$$
0 \leq|\chi|-\xi \tanh \left(\frac{\chi}{\varepsilon}\right) \leq 0.2785 \varepsilon, \quad 0 \leq \chi \tanh \left(\frac{\chi}{\varepsilon}\right)
$$

Here, RBF NNs [4, 19] are employed to approximate any continuous functions $h(Z): R^{n} \longrightarrow R$ as follows:

$$
h(Z)=\Theta^{* T} \bar{\Xi}(Z)+o(Z), \quad \forall Z \in \Pi_{Z} \subset R^{n}
$$

where $Z$ is the input vector and $n$ is the NN input dimension. $\Theta^{*}=\left[\Theta_{1}, \Theta_{2}, \ldots, \Theta_{m}\right]^{T} \in R^{m}$ is the weight vector, $m>1$ is the NN node number, and $o(Z)$ is the approximation error satisfying $|o(Z)| \leq o^{*}$ with $o^{*}>0$ as an unknown constant. $\bar{\Xi}(Z)=\left[\Xi_{1}(Z), \Xi_{2}(Z), \ldots, \Xi_{m}(Z)\right]^{T} \in R^{m}$ is the basic function vector and $\Xi_{j}(Z)$ is a commonly used Gaussian function, i.e., $\Xi_{j}(Z)=\exp \left[-\left(Z-\omega_{j}\right)^{T}\left(Z-\omega_{j}\right) / d_{j}^{2}\right], j=1,2, \ldots, m$, with center vector of $\omega_{j}=\left[\omega_{j 1}, \omega_{j 2}, \ldots, \omega_{j n}\right]^{T}$ and width of $d_{j} \in R$.

\section{Adaptive Neural PPC Controller Methodology}

In this section, a novel adaptive neural prescribed performance controller is constructed for a larger class of nonstrictfeedback nonlinear system (1) under the framework of backstepping technology. Before designing the virtual control functions, we need to first introduce the prescribed performance concept. Traditional PPC schemes [25-27, 30, 35] have certain shortcomings such as complex deductions and nondifferentiable obstacles. We attempted to resolve those shortcomings in this study via a novel asymmetry prescribed performance form:

$$
\Psi_{j}(t)<e_{j, 1}(t)<\Omega_{j}(t)
$$

where $\Omega_{j}(t)=\left(\tau_{\Omega_{j}}-\tau_{j, \infty}\right) e^{-\aleph_{\Omega_{j}} t}+\tau_{j, \infty}$ and $\Psi_{j}(t)=\left(\tau_{\Psi_{j}}+\right.$ $\left.\tau_{j, \infty}\right) e^{-\aleph_{\Psi_{j}} t}-\tau_{j, \infty}$ with $\tau_{\Omega_{j}}$ and $\tau_{\Psi_{j}}$ being the initial values of boundary functions $\Omega_{j}(t)$ and $\Psi_{j}(t)$, respectively. The design parameters $\aleph_{\Omega_{j}}>0$ and $\aleph_{\Psi_{j}}>0$ are convergence rates of exponential functions. $\Omega_{j}(\infty)=-\Psi_{j}(\infty)=\tau_{j, \infty}>0 . \tau_{\Omega_{j}}$, $\tau_{\Psi_{j}}$, and $\tau_{j, \infty}$ are properly chosen constants satisfying $\Psi_{j}(0)<$ $e_{j, 1}(0)<\Omega_{j}(0)$ and $\Psi_{j}(t)<\Omega_{j}(t)$. To this effect, $\tau_{\Omega_{j}}$ and $\tau_{\Psi_{j}}$ limit the maximum overshoot of tracking error $e_{j, 1}(t)$ at the transient.

The term $e_{j, 1}(t)$ in (6) cannot be utilized directly to construct controllers, so we introduce the novel transformed error $v_{j, 1}(t)$ :

$$
v_{j, 1}(t)=\frac{\lambda_{j, 1}(t)}{1-\lambda_{j, 1}^{2}(t)}
$$

with the normalized error

$$
\lambda_{j, 1}(t)=\frac{2 e_{j, 1}(t)-\left(\Omega_{j}(t)+\Psi_{j}(t)\right)}{\Omega_{j}(t)-\Psi_{j}(t)}
$$

where $e_{j, 1}(t)=y_{j}(t)-r_{j}(t)$. The time derivative of $v_{j, 1}(t)$ can be given by

$$
\dot{v}_{j, 1}(t)=\omega_{j, 1}\left(\dot{e}_{j, 1}(t)+\iota_{j, 1}\right)
$$

where $\omega_{j, 1}=2\left(1+\lambda_{j, 1}^{2}(t)\right) /\left(1-\lambda_{j, 1}^{2}(t)\right)^{2}\left(\Omega_{j}(t)-\Psi_{j}(t)\right)$ and $\iota_{j, 1}=\left(\lambda_{j, 1}(t)\left(\dot{\Omega}_{j}(t)-\dot{\Psi}_{j}(t)\right)-\left(\dot{\Omega}_{j}(t)+\dot{\Psi}_{j}(t)\right)\right) / 2$.

Lemma 9. For $\forall t \geq 0$, if $\Psi_{j}(0)<e_{j, 1}(0)<\Omega_{j}(0)$ and there exists a constant $\bar{v}_{j, 1}>0$ satisfying $\left|\nu_{j, 1}(t)\right| \leq \bar{\nu}_{j, 1}$, the tracking error satisfies $\Psi_{j}(t)<e_{j, 1}(t)<\Omega_{j}(t)$.

Proof. See the Appendix.

Then, the design procedure is given in a step-by-step way as follows.

Step 1. Considering the standard backstepping control design method, we define

$$
\begin{aligned}
& z_{j, 1}=v_{j, 1}, \\
& z_{j, 2}=\xi_{j, 2}-s_{j, 1}
\end{aligned}
$$

where $s_{j, 1}$ is a virtual control law to be designed later.

Differentiating $z_{j, 1}$ and considering (1) and (9) yield

$$
\dot{z}_{j, 1}=\omega_{j, 1} Q_{j, 1}+\omega_{j, 1}\left(\theta_{j, 1}\left(\xi_{j, 1}\right) \xi_{j, 2}+D_{j, 1}(\xi, t)\right)
$$

where $Q_{j, 1}=h_{j, 1}\left(\bar{\xi}_{j, \rho_{j}}\right)-\dot{r}_{j}+\iota_{j, 1}$.

Since $Q_{j, 1}$ is an unknown continuous function, we use a RBF NNs $\Theta_{j, 1}^{*}{ }^{T} \Xi_{j, 1}\left(\bar{\xi}_{j, \rho_{j}}\right)$ to approximate the function:

$$
Q_{j, 1}=\Theta_{j, 1}^{* T} \bar{\Xi}_{j, 1}\left(\bar{\xi}_{j, \rho_{j}}\right)+o_{j, 1}, \quad \forall \bar{\xi}_{j, \rho_{j}} \in \Pi_{\bar{\xi}_{j, \rho_{j}}}
$$


where $\bar{\Xi}_{j, 1}\left(\bar{\xi}_{j, \rho_{j}}\right)=\left[\Xi_{j, 1,1}\left(\bar{\xi}_{j, \rho_{j}}\right), \Xi_{j, 1,2}\left(\bar{\xi}_{j, \rho_{j}}\right), \ldots, \Xi_{j, 1, \ell_{j, 1}}\left(\bar{\xi}_{j, \rho_{j}}\right)\right]^{T}$ with $\Xi_{j, 1, n}\left(\bar{\xi}_{j, \rho_{j}}\right)$ being Gaussian functions for $n=1,2$, $\ldots, \ell_{j, 1}$, and $o_{j, 1}$ is the approximation error, satisfying $\left|o_{j, 1}\right| \leq o_{j, 1}^{*}$ with $o_{j, 1}^{*}>0$ being an unknown constant.

Consider the following quadratic Lyapunov function candidate:

$$
V_{z_{j, 1}}=\frac{1}{2} z_{j, 1}^{2}
$$

According to (11) and (12), the time derivative of (13) can be given by

$$
\begin{aligned}
\dot{V}_{z_{j, 1}}= & z_{j, 1}{\omega_{j, 1}}\left(\Theta_{j, 1}^{*} \bar{\Xi}_{j, 1}\left(\bar{\xi}_{j, \rho_{j}}\right)+o_{j, 1}\right) \\
& +z_{j, 1} \varrho_{j, 1} D_{j, 1}(\xi, t)+z_{j, 1} \Phi_{j, 1}\left(\theta_{j, 1}\left(\xi_{j, 1}\right) \xi_{j, 2}\right)
\end{aligned}
$$

Define a compact set $\Pi_{j, 1}:=\left\{z_{j, 1} \mid z_{j, 1}^{2} \leq 2 \zeta\right\}$, with $\zeta>0$ being a design constant. For the compact set $\Pi_{j, 1} \times \Pi_{j 0}$, the following lemma holds.

Lemma 10. The function $\theta_{j, 1}\left(\xi_{j, 1}\right)$ has a maximum and a minimum in $\Pi_{j, 1} \times \Pi_{j 0}$; namely, there exist constants $\underline{\theta}_{j, 1}>0$ and $\bar{\theta}_{j, 1}>0$ such that $\underline{\theta}_{j, 1}=\min _{\Pi_{j, 1} \times \Pi_{j 0}} \theta_{j, 1}\left(\xi_{j, 1}\right)$ and $\bar{\theta}_{j, 1}=$ $\max _{\Pi_{j, 1} \times \Pi_{j 0}} \theta_{j, 1}\left(\xi_{j, 1}\right)$.

Proof. See the Appendix.

Design the virtual controller law $s_{j, 1}$ as

$$
\begin{aligned}
s_{j, 1}= & -c_{j, 1} z_{j, 1} ळ_{j, 1}-\frac{\widehat{\vartheta}_{j, 1} z_{j, 1} ळ_{j, 1}}{2 b_{j, 1}^{2}} \\
& -\widehat{\psi}_{j, 1} \tanh \left(\frac{z_{j, 1} \varpi_{j, 1}}{\gamma_{j, 1}}\right)
\end{aligned}
$$

where $c_{j, 1}>0$ and $\gamma_{j, 1}>0$ are design parameters.

The corresponding parameters adaptation laws $\widehat{\vartheta}_{j, 1}$ and $\widehat{\psi}_{j, 1}$ are given by

$$
\begin{aligned}
& \dot{\hat{\vartheta}}_{j, 1}=\mu_{j, 1}\left[\frac{\left(z_{j, 1} \varpi_{j, 1}\right)^{2}}{2 b_{j, 1}^{2}}-\sigma_{j, 1} \widehat{\vartheta}_{j, 1}\right] \\
& \dot{\hat{\psi}}_{j, 1}=\rho_{j, 1}\left[z_{j, 1} \varpi_{j, 1} \tanh \left(\frac{z_{j, 1} \Phi_{j, 1}}{\gamma_{j, 1}}\right)-\sigma_{j, 1} \widehat{\psi}_{j, 1}\right]
\end{aligned}
$$

where $\mu_{j, 1}>0, \rho_{j, 1}>0$, and $\sigma_{j, 1}>0$ are design parameters; $\widehat{\vartheta}_{j, 1}$ and $\widehat{\psi}_{j, 1}$ are estimates of the unknown constants $\vartheta_{j, 1}=$ $\underline{\theta}_{j, 1}^{-1}\left\|\Theta_{j, 1}^{*}\right\|^{2} \ell_{j, 1}$ and $\psi_{j, 1}^{*}=\underline{\theta}_{j, 1}^{-1}\left(o_{j, 1}^{*}+D_{j, 1}^{*}\right)$, respectively, with $\ell_{j, 1}$ being the dimension of $\bar{\Xi}_{j, 1}\left(\bar{\chi}_{j, \rho_{j}}\right)$. According to Lemma 7, we have $\widehat{\vartheta}_{j, 1}(t) \geq 0, \widehat{\psi}_{j, 1}(t) \geq 0$ for $\forall t \geq 0$ after selecting $\widehat{\vartheta}_{j, 1}(0) \geq 0$ and $\widehat{\psi}_{j, 1}(0) \geq 0$.

In view of Young's inequality, we have

$$
\begin{aligned}
& z_{j, 1}{\Phi_{j, 1}}_{j, 1}^{* T} \bar{\Xi}_{j, 1}\left(\bar{\xi}_{j, \rho_{j}}\right) \\
& \quad \leq \frac{b_{j, 1}^{2}}{2}+\frac{\left(z_{j, 1} \oplus_{j, 1}\right)^{2}\left\|\Theta_{j, 1}^{*}\right\|^{2}}{2 b_{j, 1}^{2}} \bar{\Xi}_{j, 1}^{T}\left(\bar{\xi}_{j, \rho_{j}}\right) \bar{\Xi}_{j, 1}\left(\bar{\xi}_{j, \rho_{j}}\right)
\end{aligned}
$$

where $b_{j, 1}$ is any positive constant. From [22], we have $\bar{\Xi}_{j, 1}^{T}\left(\bar{\xi}_{j, \rho_{j}}\right) \bar{\Xi}_{j, 1}\left(\bar{\xi}_{j, \rho_{j}}\right) \leq \ell_{j, 1}$ since $\bar{\Xi}_{j, 1}\left(\bar{\xi}_{j, \rho_{j}}\right)=\left[\Xi_{j, 1,1}\left(\bar{\xi}_{j, \rho_{j}}\right)\right.$, $\left.\Xi_{j, 1,2}\left(\bar{\xi}_{j, \rho_{j}}\right), \ldots, \Xi_{j, 1, \ell_{j, 1}}\left(\bar{\xi}_{j, \rho_{j}}\right)\right]^{T}$ and $\left|\Xi_{j, 1, n}\left(\bar{\xi}_{j, \rho_{j}}\right)\right| \leq 1, n=$ $1,2, \ldots, \ell_{j, 1}$. Thus, we further have

$$
\begin{aligned}
& z_{j, 1}{\Phi_{j, 1}}_{j, 1}^{* T} \bar{\Xi}_{j, 1}\left(\bar{\xi}_{j, \rho_{j}}\right) \\
& \quad \leq \frac{\left(z_{j, 1}{\omega_{j, 1}}^{2}\left\|\Theta_{j, 1}^{*}\right\|^{2} \ell_{j, 1}\right.}{2 b_{j, 1}^{2}}+\frac{b_{j, 1}^{2}}{2}
\end{aligned}
$$

Considering (14) and (19), we obtain the time derivative of $V_{s_{j, 1}}$ as

$$
\begin{aligned}
\dot{V}_{z_{j, 1}} \leq & \frac{\left(z_{j, 1} \Phi_{j, 1}\right)^{2}\left\|\Theta_{j, 1}^{*}\right\|^{2} \ell_{j, 1}}{2 b_{j, 1}^{2}}+\frac{b_{j, 1}^{2}}{2} \\
& +z_{j, 1} \Phi_{j, 1} \theta_{j, 1}\left(\xi_{j, 1}\right) \chi_{j, 2}+\left|z_{j, 1} \Phi_{j, 1}\right| \underline{\theta}_{j, 1} \psi_{j, 1}^{*}
\end{aligned}
$$

where $\psi_{j, 1}^{*}=\underline{\theta}_{j, 1}^{-1}\left(o_{j, 1}^{*}+D_{j, 1}^{*}\right)$.

Let the Lyapunov function candidate be

$$
V_{j, 1}=V_{z_{j, 1}}+\frac{\underline{\theta}_{j, 1} \widetilde{\vartheta}_{j, 1}^{2}}{2 \mu_{j, 1}}+\frac{\underline{\theta}_{j, 1} \widetilde{\psi}_{j, 1}^{2}}{2 \rho_{j, 1}}
$$

where $\widetilde{\vartheta}_{j, 1}=\vartheta_{j, 1}-\widehat{\vartheta}_{j, 1}$ and $\widetilde{\psi}_{j, 1}=\psi_{j, 1}^{*}-\widehat{\psi}_{j, 1}$ are the estimation errors of $\vartheta_{j, 1}$ and $\psi_{j, 1}$, respectively.

From (15), (20) and $\chi_{j, 2}=z_{j, 2}+s_{j, 1}$, the time derivative of (21) is

$$
\begin{aligned}
\dot{V}_{j, 1} \leq & z_{j, 1} z_{j, 2} \Phi_{j, 1} \theta_{j, 1}\left(\xi_{j, 1}\right)+\left|z_{j, 1} \Phi_{j, 1}\right| \underline{\theta}_{j, 1} \psi_{j, 1}^{*} \\
& -c_{j, 1} \underline{\theta}_{j, 1}\left(z_{j, 1} \Phi_{j, 1}\right)^{2} \\
& -\widehat{\psi}_{j, 1} \underline{\theta}_{j, 1} z_{j, 1} \Phi_{j, 1} \tanh \left(\frac{z_{j, 1} \Phi_{j, 1}}{\gamma_{j, 1}}\right) \\
& -\frac{\underline{\theta}_{j, 1} \widehat{\vartheta}_{j, 1}\left(z_{j, 1} \Phi_{j, 1}\right)^{2}}{2 b_{j, 1}^{2}}+\frac{b_{j, 1}^{2}}{2}-\frac{\underline{\theta}_{j, 1} \widetilde{\psi}_{j, 1} \dot{\hat{\psi}}_{j, 1}}{\rho_{j, 1}} \\
& -\frac{\underline{\theta}_{j, 1} \widetilde{\vartheta}_{j, 1} \dot{\hat{\vartheta}}_{j, 1}}{\mu_{j, 1}}+\frac{\left(z_{j, 1} \Phi_{j, 1}\right)^{2}\left\|\Theta_{j, 1}^{*}\right\|^{2} \ell_{j, 1}}{2 b_{j, 1}^{2}}
\end{aligned}
$$

Substituting (16) and (17) into (22) yields

$$
\begin{aligned}
\dot{V}_{j, 1} \leq & z_{j, 1} z_{j, 2} \Phi_{j, 1} \theta_{j, 1}\left(\xi_{j, 1}\right)+\underline{\theta}_{j, 1} \sigma_{j, 1} \widetilde{\vartheta}_{j, 1} \widehat{\vartheta}_{j, 1} \\
& +\underline{\theta}_{j, 1} \sigma_{j, 1} \widetilde{\psi}_{j, 1} \widehat{\psi}_{j, 1}+0.2785 \underline{\theta}_{j, 1} \psi_{j, 1}^{*} \gamma_{j, 1} \\
& -c_{j, 1} \underline{\theta}_{j, 1}\left(z_{j, 1} \Theta_{j, 1}\right)^{2}+\frac{b_{j, 1}^{2}}{2}
\end{aligned}
$$

Step $\left(j, i_{j}\left(2 \leq i_{j} \leq \rho_{j}-1, j=1, \ldots, m\right)\right)$. In the $j, i_{j}$ th step, we define

$$
\begin{aligned}
z_{j, i_{j}} & =\xi_{j, i_{j}}-s_{j, i_{j}-1}, \\
z_{j, i_{j}+1} & =\xi_{j, i_{j}+1}-s_{j, i_{j}},
\end{aligned}
$$


where $s_{j, i_{j}-1}$ and $s_{j, i_{j}}$ are the virtual control laws of the $j, i_{j}-$ 1 th step and the $j, i_{j}$ th step, respectively.

Considering (1) and differentiating $z_{j, i_{j}}$, one has

$$
\dot{z}_{j, i_{j}}=Q_{j, i_{j}}+\theta_{j, i_{j}}\left(\bar{\xi}_{j, i_{j}}\right) \xi_{j, i_{j}+1}+D_{j, i_{j}}(\xi, t)
$$

where $Q_{j, i_{j}}=h_{j, i_{j}}\left(\bar{\xi}_{j, \rho_{j}}\right)-\dot{s}_{j, i_{j}-1}$.

Similarly, a $\operatorname{RBF}$ NNs $\Theta_{j, i_{j}}^{*}{ }^{T} \bar{\Xi}_{j, i_{j}}\left(\bar{\xi}_{j, \rho_{j}}\right)$ is employed to approximate the functions $Q_{j, i_{j}}$ :

$$
Q_{j, i_{j}}=\Theta_{j, i_{j}}^{*} T \bar{\Xi}_{j, i_{j}}\left(\bar{\xi}_{j, \rho_{j}}\right)+o_{j, i_{j}}, \quad \bar{\xi}_{j, \rho_{j}} \in \Pi_{\bar{\xi}_{j, \rho_{j}}}
$$

with $\bar{\Xi}_{j, i_{j}}\left(\bar{\xi}_{j, \rho_{j}}\right)=\left[\Xi_{j, i_{j}, 1}\left(\bar{\xi}_{j, \rho_{j}}\right), \Xi_{j, i_{j}, 2}\left(\bar{\xi}_{j, \rho_{j}}\right), \ldots, \Xi_{j, i_{j}, \ell_{j, i_{j}}}\left(\bar{\xi}_{j, \rho_{j}}\right)\right]^{T}$ and $o_{j, i_{j}}$ being the approximation error, satisfying $\left|o_{j, i_{j}}\right| \leq o_{j, i_{j}}^{*}$.

Consider the following quadratic Lyapunov function candidate:

$$
V_{z_{j, i_{j}}}=\frac{1}{2} z_{j, i_{j}}^{2}
$$

It follows from (25) and (26) that the time derivative of $V_{z_{j, i_{j}}}$ is

$$
\begin{aligned}
\dot{V}_{z_{j, i_{j}}}= & z_{j, i_{j}}\left(\theta_{j, i_{j}}\left(\bar{\xi}_{j, i_{j}}\right) \xi_{j, i_{j}+1}+o_{j, i_{j}}\right)+z_{j, i_{j}} D_{j, i_{j}}(\xi, t) \\
& +z_{j, i_{j}}\left(\Theta_{j, i_{j}}^{*} \bar{\Xi}_{j, i_{j}}\left(\bar{\xi}_{j, \rho_{j}}\right)\right)
\end{aligned}
$$

Design the virtual control laws $s_{j, i_{j}}$ as

$$
s_{j, i_{j}}=-c_{j, i_{j}} z_{j, i_{j}}-\frac{\widehat{\vartheta}_{j, i_{j}} z_{j, i_{j}}}{2 b_{j, i_{j}}^{2}}-\widehat{\psi}_{j, i_{j}} \tanh \left(\frac{z_{j, i_{j}}}{\gamma_{j, i_{j}}}\right)
$$

The design process of parameters is similar to Step 1, and the parameters adaptation laws $\widehat{\vartheta}_{j, i_{j}}$ and $\widehat{\psi}_{j, i_{j}}$ are given by

$$
\begin{aligned}
& \dot{\widehat{\vartheta}}_{j, i_{j}}=\mu_{j, i_{j}}\left[\frac{z_{j, i_{j}}^{2}}{2 b_{j, i_{j}}^{2}}-\sigma_{j, i_{j}} \widehat{\vartheta}_{j, i_{j}}\right] \\
& \dot{\widehat{\psi}}_{j, i_{j}}=\rho_{j, i_{j}}\left[z_{j, i_{j}} \tanh \left(\frac{z_{j, i_{j}}}{\gamma_{j, i_{j}}}\right)-\sigma_{j, i_{j}} \widehat{\psi}_{j, i_{j}}\right]
\end{aligned}
$$

The design process of parameters is also similar to Step 1. The functions $\theta_{j, i_{j}}\left(\bar{\xi}_{j, i_{j}}\right)$ can be rewritten as

$$
\theta_{j, i_{j}}\left(\bar{\xi}_{j, i_{j}}\right)=\Phi_{j, i_{j}}\left(\bar{z}_{j, i_{j}}, \overline{\widehat{\vartheta}}_{j, i_{j}-1}, \overline{\widehat{\psi}}_{j, i_{j}-1}, r_{j}\right)
$$

where $\bar{z}_{j, i_{j}}=\left[z_{j, 1}, z_{j, 2}, \ldots, z_{j, i_{j}}\right]^{T}, \overline{\widehat{\vartheta}}_{j, i_{j}-1}=\left[\widehat{\vartheta}_{j, 1}, \widehat{\vartheta}_{j, 2}, \ldots\right.$, $\left.\widehat{\vartheta}_{j, i_{j}-1}\right]^{T}, \widehat{\psi}_{j, i_{j}-1}=\left[\widehat{\psi}_{j, 1}, \widehat{\psi}_{j, 2}, \ldots \widehat{\psi}_{j, i_{j}-1}\right]^{T}$, and $\Phi_{j, i_{j}}(\cdot)$ is a continuous function.
Define the compact sets $\Pi_{j, i_{j}}$ as

$$
\begin{aligned}
\Pi_{j, i_{j}} & :=\left\{\left(\bar{z}_{j, i_{j}}^{T}, \overline{\widehat{\vartheta}}_{j, i_{j}-1}^{T}, \overline{\widehat{\psi}}_{j, i_{j}-1}^{T}\right)^{T} \mid \sum_{c=1}^{i_{j}}\left(z_{j, c}^{2}\right)\right. \\
+ & \left.\sum_{c=1}^{i_{j}-1}\left(\frac{\underline{\theta}_{j, c} \widetilde{\vartheta}_{j, c}^{2}}{\mu_{j, c}}+\frac{\underline{\theta}_{j, c} \widetilde{\psi}_{j, c}^{2}}{\rho_{j, c}}\right) \leq 2 \zeta\right\}
\end{aligned}
$$

where $\zeta>0$. Similarly, we can know that the functions $\theta_{j, i_{j}}\left(\bar{\xi}_{j, i_{j}}\right)$ have a maximum and a minimum in the compact set $\Pi_{j, i_{j}} \times \Pi_{j 0}$; namely, there exist constants $\bar{\theta}_{j, i_{j}}>0$ and $\underline{\theta}_{j, i_{j}}>0$ satisfying

$$
0<\underline{\theta}_{j, i_{j}} \leq \theta_{j, i_{j}}\left(\bar{\xi}_{j, i_{j}}\right) \leq \bar{\theta}_{j, i_{j}}, \quad \bar{\xi}_{j, i_{j}} \in \Pi_{j, i_{j}} \times \Pi_{j 0}
$$

Let the Lyapunov function candidate be

$$
V_{j, i_{j}}=V_{z_{j, i_{j}}}+\frac{\underline{\theta}_{j, i_{j}} \widetilde{\vartheta}_{j, i_{j}}^{2}}{2 \mu_{j, i_{j}}}+\frac{\underline{\theta}_{j, i_{j}} \widetilde{\psi}_{j, i_{j}}^{2}}{2 \rho_{j, i_{j}}}
$$

where $\widetilde{\vartheta}_{j, i_{j}}=\vartheta_{j, i_{j}}-\widehat{\vartheta}_{j, i_{j}}$ and $\widetilde{\psi}_{j, i_{j}}=\psi_{j, i_{j}}^{*}-\widehat{\psi}_{j, i_{j}}$.

According to Young's inequality, one has

$$
z_{j, i_{j}} \Theta_{j, i_{j}}^{* T} \bar{\Xi}_{j, i_{j}}\left(\bar{\xi}_{j, \rho_{j}}\right) \leq \frac{z_{j, i_{j}}^{2}\left\|\Theta_{j, i_{j}}^{*}\right\|^{2} \ell_{j, i_{j}}}{2 b_{j, i_{j}}^{2}}+\frac{b_{j, i_{j}}^{2}}{2}
$$

where $b_{j, i_{j}}$ is any positive constant. $\ell_{j, i_{j}}$ is the dimension of $\bar{\Xi}_{j, i_{j}}\left(\bar{\xi}_{j, \rho_{j}}\right)=\left[\Xi_{j, i_{j}, 1}\left(\bar{\xi}_{j, \rho_{j}}\right), \Xi_{j, i_{j}, 2}\left(\bar{\xi}_{j, \rho_{j}}\right), \ldots, \Xi_{j, i_{j}, \ell_{j, i_{j}}}\left(\bar{\xi}_{j, \rho_{j}}\right)\right]^{T}$ with $\left|\Xi_{j, i_{j}, n}\left(\bar{\xi}_{j, \rho_{j}}\right)\right| \leq 1$, for $n=1,2, \ldots, \ell_{j, i_{j}}$

From (36), we can rewrite (28) as

$$
\begin{aligned}
\dot{V}_{z_{j, i_{j}}} \leq & z_{j, i_{j}} \theta_{j, i_{j}}\left(\bar{\xi}_{j, i_{j}}\right) \xi_{j, i_{j}+1}+\frac{z_{j, i_{j}}^{2}\left\|\Theta_{j, i_{j}}^{*}\right\|^{2} \ell_{j, i_{j}}}{2 b_{j, i_{j}}^{2}}+\frac{b_{j, i_{j}}^{2}}{2} \\
& +\left|z_{j, i_{j}}\right| \underline{\theta}_{j, i_{j}} \psi_{j, i_{j}}^{*}
\end{aligned}
$$

where $\psi_{j, i_{j}}^{*}=\underline{\theta}_{j, i_{j}}^{-1}\left(o_{j, i_{j}}^{*}+D_{j, i_{j}}^{*}\right)$.

According to (29), (30), (31), and (37), we obtain the time derivative of $V_{j, i_{j}}$ as

$$
\begin{gathered}
\dot{V}_{j, i_{j}} \leq z_{j, i_{j}} z_{j, i_{j}+1} \theta_{j, i_{j}}\left(\bar{\xi}_{j, i_{j}}\right)+\underline{\theta}_{j, i_{j}} \sigma_{j, i_{j}} \widetilde{\vartheta}_{j, i_{j}} \widehat{\vartheta}_{j, i_{j}} \\
+\underline{\theta}_{j, i_{j}} \sigma_{j, i_{j}} \widetilde{\psi}_{j, i_{j}} \widehat{\psi}_{j, i_{j}}-c_{j, i_{j}} \underline{\theta}_{j, i_{j}} z_{j, i_{j}}^{2} \\
+0.2785 \underline{\theta}_{j, i_{j}} \psi_{j, i_{j}}^{*} \gamma_{j, i_{j}}+\frac{b_{j, i_{j}}^{2}}{2}
\end{gathered}
$$

Step $\left(j, \rho_{j}(j=1, \ldots, m)\right)$. Define $z_{j, \rho_{j}}=\xi_{j, \rho_{j}}-s_{j, \rho_{j}-1}-$ $\hbar_{j} \tanh \beta_{j}$, with $\hbar_{j}>0$ being a design parameter, and $\beta_{j}$ is defined as

$$
\dot{\beta}_{j}=\frac{\cosh ^{2} \beta_{j}}{\hbar_{j}}\left(-\alpha_{j} \tanh \beta_{j}+\operatorname{sat}\left(\iota_{j}\right)-\iota_{j}\right),
$$

$$
\beta_{j}(0)=0
$$

where $\alpha_{j}>0$ is a design parameter. 
Considering (1) and differentiating $z_{j, \rho_{j}}$ with respect to time yields

$$
\begin{aligned}
\dot{z}_{j, \rho_{j}}= & Q_{j, \rho_{j}}+\theta_{j, \rho_{j}}\left(\bar{\xi}_{j, \rho_{j}}\right) u_{j}+D_{j, \rho_{j}}(\xi, t) \\
& +\alpha_{j} \tanh \beta_{j}-\operatorname{sat}\left(\iota_{j}\right)+\iota_{j}
\end{aligned}
$$

where $Q_{j, \rho_{j}}=h_{j, \rho_{j}}\left(\bar{\xi}_{j, \rho_{j}}\right)-s_{j, \rho_{j}-1}$. For $\left|u_{j}\right|=\left|\operatorname{sat}\left(\iota_{j}\right)\right| \leq u_{j, M}$, there exists a continuous function $G_{j, \rho_{j}}\left(\bar{\xi}_{j, \rho_{j}}\right)$ such that

$$
\left|\theta_{j, \rho_{j}}\left(\bar{\xi}_{j, \rho_{j}}\right) u_{j}\right|=\left|\theta_{j, \rho_{j}}\left(\bar{\xi}_{j, \rho_{j}}\right) \operatorname{sat}\left(\iota_{j}\right)\right| \leq G_{j, \rho_{j}}\left(\bar{\xi}_{j, \rho_{j}}\right)
$$

Define the following compact set

$$
\begin{gathered}
\Pi_{j, \rho_{j}}:=\left\{\left(\bar{z}_{j, \rho_{j}}^{T}, \overline{\widehat{\vartheta}}_{j, \rho_{j}-1}^{T}, \overline{\widehat{\psi}}_{j, \rho_{j}-1}^{T}\right)^{T} \mid \sum_{c=1}^{\rho_{j}}\left(z_{j, c}^{2}\right)\right. \\
\left.+\sum_{c=1}^{\rho_{j}-1}\left(\frac{\underline{\theta}_{j, c} \widetilde{\vartheta}_{j, c}^{2}}{\mu_{j, c}}+\frac{\underline{\theta}_{j, c} \widetilde{\psi}_{j, c}^{2}}{\rho_{j, c}}\right) \leq 2 \zeta\right\}
\end{gathered}
$$

The functions $G_{j, \rho_{j}}\left(\bar{\xi}_{j, \rho_{j}}\right)=G_{j, \rho_{j}}\left(\bar{\xi}_{j, \rho_{j}-1}, z_{j, \rho_{j}}+s_{j, \rho_{j}-1}\right)$ have maximums on $\Pi_{j, \rho_{j}} \times \Pi_{j 0}$. Thus, we have $G_{j, \rho_{j}}\left(\bar{\xi}_{j, \rho_{j}}\right) \leq G_{j, \rho_{j}}^{*}$ with $G_{j, \rho_{j}}^{*}>0$ being the unknown constants.

Choose the actual control law $\iota_{j}$ as

$$
\iota_{j}=-c_{j, \rho_{j}} z_{j, \rho_{j}}-\frac{\widehat{\vartheta}_{j, \rho_{j}} z_{j, \rho_{j}}}{2 b_{j, \rho_{j}}^{2}}-\widehat{\psi}_{j, \rho_{j}} \tanh \left(\frac{z_{j, \rho_{j}}}{\gamma_{j, \rho_{j}}}\right)
$$

The design process of parameters is similar to step $j, i_{j}$ and Step 1, and the parameters adaptation laws $\widehat{\vartheta}_{j, \rho_{j}}$ and $\widehat{\psi}_{j, \rho_{j}}$ are given by

$$
\begin{aligned}
& \dot{\widehat{\vartheta}}_{j, \rho_{j}}=\mu_{j, \rho_{j}}\left[\frac{z_{j, \rho_{j}}^{2}}{2 b_{j, \rho_{j}}^{2}}-\sigma_{j, \rho_{j}} \widehat{\vartheta}_{j, \rho_{j}}\right] \\
& \dot{\hat{\psi}}_{j, \rho_{j}}=\rho_{j, \rho_{j}}\left[z_{j, \rho_{j}} \tanh \left(\frac{z_{j, \rho_{j}}}{\gamma_{j, \rho_{j}}}\right)-\sigma_{j, \rho_{j}} \widehat{\psi}_{j, \rho_{j}}\right]
\end{aligned}
$$

where $\psi_{j, \rho_{j}}^{*}=o_{j, \rho_{j}}^{*}+D_{j, \rho_{j}}^{*}+G_{j, \rho_{j}}^{*}+\alpha_{j}+u_{j, M}$, and the design process of other parameters is also similar to step $j, i_{j}$ and Step 1.

Similar to the former steps, the function $\theta_{j, \rho_{j}}\left(\bar{\xi}_{j, \rho_{j}}\right)$ can be expressed as

$$
\theta_{j, \rho_{j}}\left(\bar{\xi}_{j, \rho_{j}}\right)=\Phi_{j, \rho_{j}}\left(\bar{z}_{j, \rho_{j}}, \overline{\widehat{\vartheta}}_{j, \rho_{j}-1}, \overline{\widehat{\psi}}_{j, \rho_{j}-1}, r_{j}\right)
$$

with $\Phi_{j, \rho_{j}}(\cdot)$ being a continuous function.

In line with Lemma 10 , the function $\Phi_{j, \rho_{j}}(\cdot)$ has maxi$\operatorname{mum} \bar{\theta}_{j, \rho_{j}}$ and minimum $\underline{\theta}_{j, \rho_{j}}$ such that

$$
0<\underline{\theta}_{j, \rho_{j}} \leq \theta_{j, \rho_{j}}\left(\bar{\xi}_{j, \rho_{j}}\right) \leq \bar{\theta}_{j, \rho_{j}}
$$

Similarly to the previous steps, define $V_{z_{j, \rho_{j}}}=z_{j, \rho_{j}}^{2} / 2$, and a RBF NNs $\Theta_{j, \rho_{j}}^{*}{ }^{T} \bar{\Xi}_{j, \rho_{j}}\left(\bar{\chi}_{j, \rho_{j}}\right)$ is utilized to approximate the functions $Q_{j, \rho_{j}}$. According to (40), (41), and (47), the time derivative of $V_{z_{j, \rho_{j}}}$ is

$$
\dot{V}_{z_{j, \rho_{j}}} \leq \frac{z_{j, \rho_{j}}^{2}\left\|\Theta_{j, \rho_{j}}^{*}\right\|^{2} \ell_{j, \rho_{j}}}{2 b_{j, \rho_{j}}^{2}}+\frac{b_{j, \rho_{j}}^{2}}{2}+\left|z_{j, \rho_{j}}\right| \psi_{j, \rho_{j}}^{*}+\iota_{j} s_{j, \rho_{j}}
$$

where $b_{j, \rho_{j}}>0$ and $\psi_{j, \rho_{j}}^{*}=o_{j, \rho_{j}}^{*}+D_{j, \rho_{j}}^{*}+G_{j, \rho_{j}}^{*}+\alpha_{j}+u_{j, M}$.

Consider the Lyapunov function candidate as

$$
V_{j, \rho_{j}}=V_{z_{j, \rho_{j}}}+\frac{\widetilde{\vartheta}_{j, \rho_{j}}^{2}}{2 \mu_{j, \rho_{j}}}+\frac{\widetilde{\psi}_{j, \rho_{j}}^{2}}{2 \rho_{j, \rho_{j}}}
$$

where $\widetilde{\vartheta}_{j, \rho_{j}}=\vartheta_{j, \rho_{j}}-\widehat{\vartheta}_{j, \rho_{j}}$ and $\widetilde{\psi}_{j, \rho_{j}}=\psi_{j, \rho_{j}}^{*}-\widehat{\psi}_{j, \rho_{j}}$.

Substituting (43), (44), (45), and (48) into (49), the time derivative of $V_{j, \rho_{j}}$ is

$$
\begin{aligned}
\dot{V}_{j, \rho_{j}} \leq & \sigma_{j, \rho_{j}} \widetilde{\vartheta}_{j, \rho_{j}} \widehat{\vartheta}_{j, \rho_{j}}+\sigma_{j, \rho_{j}} \widetilde{\psi}_{j, \rho_{j}} \widehat{\psi}_{j, \rho_{j}}-c_{j, \rho_{j}} z_{j, \rho_{j}}^{2} \\
& +0.2785 \psi_{j, \rho_{j}}^{*} \gamma_{j, \rho_{j}}+\frac{b_{j, \rho_{j}}^{2}}{2}
\end{aligned}
$$

\section{Stability Analysis}

Choose the Lyapunov function as follows:

$$
V=\sum_{j=1}^{m} V_{j}
$$

where $V_{j}$ is the Lyapunov function for the $j$ th subsystem

$$
\begin{aligned}
V_{j}= & \frac{1}{2} \sum_{i_{j}=1}^{\rho_{j}-1}\left(+\frac{\underline{\theta}_{j, i_{j}} \widetilde{\vartheta}_{j, i_{j}}^{2}}{\mu_{j, i_{j}}}+\frac{\underline{\theta}_{j, i_{j}} \widetilde{\psi}_{j, i_{j}}^{2}}{\rho_{j, i_{j}}}\right)+\frac{1}{2} \sum_{i_{j}=1}^{\rho_{j}}\left(z_{j, i_{j}}^{2}\right) \\
& +\frac{\widetilde{\vartheta}_{j, \rho_{j}}^{2}}{2 \mu_{j, \rho_{j}}}+\frac{\widetilde{\psi}_{j, \rho_{j}}^{2}}{2 \rho_{j, \rho_{j}}}
\end{aligned}
$$

The main stability result of the proposed scheme is summarized in the following Theorem 11.

Theorem 11. Consider Assumptions 1-6 and the intermediate virtual control laws (15), (29), the actual control law (43), and the adaptive laws (16), (17), (30), (31), (44), and (45). For $\Psi_{j}(0)<e_{j, 1}(0)<\Omega_{j}(0), \forall \zeta>0, \widehat{\vartheta}_{j, i_{j}}(t) \geq 0, \widehat{\psi}_{j, i_{j}}(t) \geq 0$, and $V_{j}(0) \leq \zeta$, there exist design parameters $\sigma_{j, i_{j}}, \gamma_{j, i_{j}}, b_{j, i_{j}}$, $c_{j, i_{j}}, \rho_{j, i_{j}}$, and $\mu_{j, i_{j}}$ such that

(i) all the closed-loop system signals are SGUUB;

(ii) the whole system output tracking error $e_{1}$ remains in a neighborhood of the origin within the preselected transient and steady bounds; and

(iii) the closed-loop system variable $\beta_{j}$ is bounded. 
Proof. In view of (23), (38), and (50), the time derivative of $V_{j}$ is

$$
\begin{aligned}
\dot{V}_{j} \leq & \sum_{i_{j}=1}^{\rho_{j}-1}\left(\underline{\theta}_{j, i_{j}} \sigma_{j, i_{j}} \widetilde{\vartheta}_{j, i_{j}} \widehat{\vartheta}_{j, i_{j}}+\underline{\theta}_{j, i_{j}} \sigma_{j, i_{j}} \widetilde{\psi}_{j, i_{j}} \widehat{\psi}_{j, i_{j}}\right) \\
& +\sum_{i_{j}=1}^{\rho_{j}}\left(\frac{b_{j, i_{j}}^{2}}{2}\right)+\sigma_{j, \rho_{j}} \widetilde{\vartheta}_{j, \rho_{j}} \widehat{\vartheta}_{j, \rho_{j}}+\sigma_{j, \rho_{j}} \widetilde{\psi}_{j, \rho_{j}} \widehat{\psi}_{j, \rho_{j}} \\
& -c_{j, 1} \underline{\theta}_{j, 1}\left(z_{j, 1} \Theta_{j, 1}\right)^{2}+\sum_{i_{j}=1}^{\rho_{j}-1}\left(0.2785 \underline{\theta}_{j, i_{j}} \psi_{j, i_{j}}^{*} \gamma_{j, i_{j}}\right) \\
& -\sum_{i_{j}=2}^{\rho_{j}-1}\left(c_{j, i_{j}} \theta_{j, i_{j}} z_{j, i_{j}}^{2}\right)+0.2785 \psi_{j, \rho_{j}}^{*} \gamma_{j, \rho_{j}} \\
& +z_{j, 1} z_{j, 2} \Theta_{j, 1} \theta_{j, 1}\left(\xi_{j, 1}\right) \\
& +\sum_{i_{j}=2} z_{j, i_{j}} z_{j, i_{j}+1} \theta_{j, i_{j}}\left(\bar{\xi}_{j, i_{j}}\right)-c_{j, \rho_{j}} z_{j, \rho_{j}}^{2}
\end{aligned}
$$

By completion of squares, it holds that

$$
\begin{aligned}
& \widetilde{\vartheta}_{j, i_{j}} \widehat{\vartheta}_{j, i_{j}}=\widetilde{\vartheta}_{j, i_{j}}\left(\vartheta_{j, i_{j}}-\widetilde{\vartheta}_{j, i_{j}}\right) \\
& \leq \frac{\mathcal{\vartheta}_{j, i_{j}}^{2}}{2}-\frac{\widetilde{\vartheta}_{j, i_{j}}^{2}}{2} \\
& \widetilde{\psi}_{j, i_{j}} \widehat{\psi}_{j, i_{j}}=\widetilde{\psi}_{j, i_{j}}\left(\psi_{j, i_{j}}^{*}-\widetilde{\psi}_{j, i_{j}}\right) \\
& \leq \frac{\psi_{j, i_{j}}^{* 2}}{2}-\frac{\widetilde{\psi}_{j, i_{j}}^{2}}{2} \\
& z_{j, 1} z_{j, 2} \omega_{j, 1} \theta_{j, 1}\left(\xi_{j, 1}\right) \leq \frac{\left(z_{j, 1} \varpi_{j, 1}\right)^{2} \bar{\theta}_{j, 1} k_{j, 1}}{2}+\frac{\bar{\theta}_{j, 1} z_{j, 2}^{2}}{2 k_{j, 1}} \\
& z_{j, i_{j}} z_{j, i_{j}+1} \theta_{j, i_{j}}\left(\bar{\xi}_{j, i_{j}}\right) \leq \frac{z_{j, i_{j}}^{2} \bar{\theta}_{j, i_{j}} k_{j, i_{j}}}{2}+\frac{\bar{\theta}_{j, i_{j}} z_{j, i_{j}+1}^{2}}{2 k_{j, i_{j}}}
\end{aligned}
$$

where $k_{j, 1}$ and $k_{j, i_{j}}$ are unknown positive constants.

Then, (53) can be rewritten as

$$
\begin{aligned}
\dot{V}_{j} \leq & -\sum_{i_{j}=2}^{\rho_{j}-1}\left(c_{j, i_{j}} \theta_{j, i_{j}}-\frac{\bar{\theta}_{j, i_{j}-1}}{2 k_{j, i_{j}-1}}-\frac{\bar{\theta}_{j, i_{j}} k_{j, i_{j}}}{2}\right) z_{j, i_{j}}^{2}+\Sigma_{j} \\
& -\left(c_{j, 1} \underline{\theta}_{j, 1}-\frac{\bar{\theta}_{j, 1} k_{j, 1}}{2}\right) \omega_{j, 1}^{2} z_{j, 1}^{2}-\sigma_{j, \rho_{j}} \frac{\widetilde{\psi}_{j, \rho_{j}}^{2}}{2} \\
& -\sum_{i_{j}=1}^{\rho_{j}-1}\left(\underline{\theta}_{j, i_{j}} \sigma_{j, i_{j}} \frac{\widetilde{\vartheta}_{j, i_{j}}^{2}}{2}+\underline{\theta}_{j, i_{j}} \sigma_{j, i_{j}} \frac{\widetilde{\psi}_{j, i_{j}}^{2}}{2}\right) \\
& -\left(c_{j, \rho_{j}}-\frac{\bar{\theta}_{j, \rho_{j}-1}}{2 k_{j, \rho_{j}-1}}\right) z_{j, \rho_{j}}^{2}-\sigma_{j, \rho_{j}} \frac{\widetilde{\vartheta}_{j, \rho_{j}}^{2}}{2}
\end{aligned}
$$

where $\Sigma_{j}=\sum_{i_{j}=1}^{\rho_{j}-1}\left(\underline{\theta}_{j, i_{j}} \sigma_{j, i_{j}} \vartheta_{j, i_{j}}^{2} / 2+\underline{\theta}_{j, i_{j}} \sigma_{j, i_{j}} \psi_{j, i_{j}}^{* 2} / 2+\right.$ $\left.0.2785 \underline{\theta}_{j, i_{j}} \psi_{j, i_{j}}^{*} \gamma_{j, i_{j}}\right)+\sigma_{j, \rho_{j}} \widetilde{\vartheta}_{j, \rho_{j}} \widehat{\vartheta}_{j, \rho_{j}}+\sigma_{j, \rho_{j}} \widetilde{\psi}_{j, \rho_{j}} \widehat{\psi}_{j, \rho_{j}}+\sum_{i_{j}=1}^{\rho_{j}}\left(b_{j, i_{j}}^{2} /\right.$ 2) $+0.2785 \psi_{j, \rho_{j}}^{*} \gamma_{j, \rho_{j}}$.

From ${\omega_{j, 1}}=2\left(1+\lambda_{j, 1}^{2}(t)\right) /\left(1-\lambda_{j, 1}^{2}(t)\right)^{2}\left(\Omega_{j}(t)-\Psi_{j}(t)\right)$, we can further have $2 /\left(\Omega_{j}(0)-\Psi_{j}(0)\right) \leq \Phi_{j, 1}$ on compact set $\Pi_{j, \rho_{j}} \times \Pi_{j 0}$. Considering $\omega_{j, 1}^{2} \geq 4 /\left(\Omega_{j}(0)-\Psi_{j}(0)\right)^{2}$, choose the control parameters $c_{j, 1} \geq\left[\left(\kappa_{j, 1}\left(\Omega_{j}(0)-\Psi_{j}(0)\right)^{2}\right) / 4+\right.$ $\left.\left(\bar{\theta}_{j, 1} k_{j, 1}\right) / 2\right] / \underline{\theta}_{j, 1}, c_{j, i_{j}} \geq\left[\left(\bar{\theta}_{j, i_{j}} k_{j, i_{j}}\right) / 2+\bar{\theta}_{j, i_{j}} /\left(2 k_{j, i_{j}}\right)+\kappa_{j, i_{j}}\right] /$ $\underline{\theta}_{j, i_{j}}, c_{j, \rho_{j}} \geq\left[\bar{\theta}_{j, \rho_{j}-1} /\left(2 k_{j, \rho_{j}-1}\right)+\kappa_{j, \rho_{j}}\right] / \underline{\theta}_{j, \rho_{j}}, \sigma_{j, i_{j}} \geq \kappa_{j, i_{j}} /$ $\underline{\theta}_{j, i_{j}}\left(i_{j}=1,2, \ldots, \rho_{j}-1, j=1,2, \ldots, m\right), \sigma_{j, \rho_{j}} \geq \kappa_{j, \rho_{j}}$ with $\kappa_{j, i_{j}}$ being a positive constant.

Invoking (55), we can obtain

$$
\dot{V}_{j} \leq-\kappa_{j} V_{j}+\Sigma_{j}
$$

where $\kappa_{j}=\min \left\{2 \kappa_{j, i_{i}}, \rho_{j, i_{j}} \kappa_{j, i_{j}}, \mu_{j, i_{j}} \kappa_{j, i_{j}}\right\}\left(i_{j}=1,2, \ldots, \rho_{j}\right)$.

Note that $\Sigma_{j} / \kappa_{j}$ can be made arbitrarily small by decreasing $\sigma_{j, i_{j}}, \gamma_{j, i_{j}}$, and $b_{j, i_{j}}$, increasing $c_{j, i_{j}}, \rho_{j, i_{j}}$, and $\mu_{j, i_{j}}$ in the meanwhile. Thus, we can have $\Sigma_{j} / \kappa_{j} \leq \zeta$ by selecting proper design parameters. It follows from $\Sigma_{j} / \kappa_{j} \leq \zeta$ and (56) that $\dot{V}_{j} \leq 0$ on the level set $V_{j}=\zeta$. Subsequently, the compact set $\Pi_{j, \rho_{j}} \times \Pi_{j 0}$ is an invariant set and the signals $z_{j, i_{j}}, \widetilde{\vartheta}_{j, i_{j}}$ and $\widetilde{\psi}_{j, i_{j}}\left(i_{j}=1,2, \ldots, \rho_{j}\right)$ of the closed-loop system are SGUUB. The property (i) of Theorem 11 is proved.

Multiplying (56) by $e^{\kappa_{j} t}$ and integrating over $[0, t]$ yields

$$
0 \leq V_{j}(t) \leq V_{j}(0) e^{-\kappa_{j} t}+\mathrm{T}_{j}, \quad \forall t \geq 0
$$

where $\mathrm{T}_{j}=\Sigma_{j} / \kappa_{j}$.

According to (21) and (57), we have

$$
\frac{1}{2} z_{j, 1}^{2}=\frac{1}{2} v_{j, 1}^{2} \leq V_{j}(0) e^{-\kappa_{j} t}+\mathrm{T}_{j}, \quad \forall t \geq 0
$$

From $z_{j, 1} \leq \sqrt{2\left(V_{j}(0)+\mathrm{T}_{j}\right)}, \forall t \geq 0$ and Lemma 9, we have $\Psi_{j}(t)<e_{j, 1}(t)<\Omega_{j}(t)$, for $\forall t \geq 0$. Now let us consider the Lyapunov function candidate for the whole systems as $V=\sum_{j=1}^{m} V_{j}$. From (57), it can be derived that

$$
\dot{V}=\sum_{j=1}^{m} \dot{V}_{j} \leq \sum_{j=1}^{m}\left[-\kappa_{j} V_{j}+\Sigma_{j}\right] \leq-\Lambda V+\mathrm{X}
$$

where $\Lambda=\min \left\{\kappa_{1}, \ldots, \kappa_{m}\right\}$ and $\mathrm{X}=\sum_{j=1}^{m} \Sigma_{j}$. Then, we further have

$$
V(t) \leq V(0) e^{-\Lambda t}+\Upsilon, \quad \forall t \geq 0
$$

where $Y=X / \Lambda$ is a positive constant.

Similarly, from (21) and (60), we can obtain

$$
\frac{1}{2} z_{1}^{2}=\frac{1}{2} v_{1}^{2} \leq V(0) e^{-\Lambda t}+\Upsilon, \quad \forall t \geq 0
$$

From $z_{1} \leq \sqrt{2(V(0)+\Upsilon)}, \quad \forall t \geq 0$ and Lemma 9, we have $\Psi(t)<e_{1}(t)<\Omega(t)$, for $\forall t \geq 0$. Consequently, by appropriately choosing the design parameters, the tracking control 
error can be shown to converge to a small neighborhood of the origin and its prescribed performance is satisfied. Thus, property (ii) of Theorem 11 is proved.

Furthermore, there exists a scalar $\omega_{j} \geq 0$ to satisfy $\left|\Gamma_{j}\right| \leq$ $\omega_{j}$ with $\Gamma_{j}=\operatorname{sat}\left(\iota_{j}\right)-\iota_{j}$ and $\alpha_{j}>\omega_{j}$. Define $V_{\beta_{j}}=\hbar_{j} \beta_{j}^{2} / 2$, we can have

$$
\dot{V}_{\beta_{j}} \leq \cosh ^{2} \beta_{j}\left[0.2785 \alpha_{j}-\left(\alpha_{j}-\omega_{j}\right)\left|\beta_{j}\right|\right]
$$

If $\left|\beta_{j}\right|>0.2785 \alpha_{j} /\left(\alpha_{j}-\omega_{j}\right)$, we can obtain $\dot{V}_{\beta_{j}}<0$. Thus $\beta_{j}$ will lie in the compact set $\left\{\beta_{j}|| \beta_{j} \mid \leq 0.2785 \alpha_{j} /\left(\alpha_{j}-\omega_{j}\right)\right\}$, for $\forall \mathrm{t} \geq 0$. This completes the proof.

\section{Simulation Analysis}

In this section, to illustrate the effectiveness of the proposed control scheme, two simulation examples are considered.

Example 12. Consider the following uncertain nonlinear systems

$$
\begin{aligned}
\dot{\xi}_{1,1} & =h_{1,1}\left(\bar{\xi}_{1,2}\right)+\theta_{1,1}\left(\bar{\xi}_{1,1}\right) \xi_{1,2}+D_{1,1}(\xi, t) \\
\dot{\xi}_{1,2} & =h_{1,2}\left(\bar{\xi}_{1,2}\right)+\theta_{1,2}\left(\bar{\xi}_{1,2}\right) u_{1}\left(\iota_{1}\right)+D_{1,2}(\xi, t) \\
\dot{\xi}_{2,1} & =h_{2,1}\left(\bar{\xi}_{2,2}\right)+\theta_{2,1}\left(\bar{\xi}_{2,1}\right) \xi_{2,2}+D_{2,1}(\xi, t) \\
\dot{\xi}_{2,2} & =h_{2,2}\left(\bar{\xi}_{2,2}\right)+\theta_{2,2}\left(\bar{\xi}_{2,2}\right) u_{2}\left(\iota_{2}\right)+D_{2,2}(\xi, t) \\
y_{1} & =\xi_{1,1} \\
y_{2} & =\xi_{2,1}
\end{aligned}
$$

where $h_{1,1}\left(\bar{\xi}_{1,2}\right)=\xi_{1,1} \xi_{1,2} e^{-1.5 \xi_{1,2}^{2}}, h_{1,2}\left(\bar{\xi}_{1,2}\right)=\cos \left(\xi_{1,1} \xi_{1,2}\right) \xi_{1,2}^{2}$, $h_{2,1}\left(\bar{\xi}_{2,2}\right)=\left(0.8+\sin \left(\xi_{1,2} \xi_{2,1}^{2}\right)\right), h_{2,2}\left(\bar{\xi}_{2,2}\right)=\xi_{1,1} \xi_{1,2}^{2}+$ $\xi_{2,1}^{2} \xi_{2,2}$ and $\theta_{1,1}\left(\bar{\xi}_{1,1}\right)=\left(1+e^{\xi_{1,1}^{2}}\right), \theta_{2,1}\left(\bar{\xi}_{2,1}\right)=e^{\xi_{1,1} \xi_{2,1}}$, $\theta_{1,2}\left(\bar{\xi}_{1,2}\right)=\left(1.2+e^{\xi_{1,1} \xi_{1,2}^{3}}\right), \theta_{2,2}\left(\bar{\xi}_{2,2}\right)=\left(2.5+e^{\xi_{1,2} \xi_{2,1} \xi_{2,2}}\right)$. $D_{1,1}=0.5 \cos (0.5 t) \sin \left(\xi_{1,1} \xi_{1,2}^{2} \xi_{2,1}\right), D_{2,1}=0.5 \cos \left(\xi_{1,1} \xi_{1,2}^{2} \xi_{2,2}^{2}\right)$, $D_{1,2}=2 \sin \left(\xi_{1,2}^{2}+\xi_{1,1} \xi_{2,1}\right)$, and $D_{2,2}=\cos \left(\xi_{2,2}^{2}+\xi_{2,1}^{2}\right)(\sin (t))^{2}$. The input saturations $u_{1}\left(\iota_{1}\right)$ and $u_{2}\left(l_{2}\right)$ are described by

$$
\begin{aligned}
& u_{1}\left(\iota_{1}\right)=\operatorname{sat}\left(\iota_{1}\right)= \begin{cases}\operatorname{sign}\left(\iota_{1}\right) 3.5, & \iota_{1} \geq 3.5 \\
\iota_{1}, & \iota_{1}<3.5\end{cases} \\
& u_{2}\left(\iota_{2}\right)=\operatorname{sat}\left(\iota_{2}\right)= \begin{cases}\operatorname{sign}\left(\iota_{2}\right) 4.5, & \iota_{2} \geq 4.5 \\
\iota_{2}, & \iota_{2}<4.5\end{cases}
\end{aligned}
$$

Choose the reference signal as the van der pol oscillator system described as follows:

$$
\begin{aligned}
\dot{\xi}_{r 1} & =\xi_{r 2} \\
\dot{\xi}_{r 2} & =-\xi_{r 1}+\overline{\mathrm{T}}\left(1-\xi_{r 2}^{2}\right) \xi_{r 2} \\
y_{r} & =\xi_{r 1}
\end{aligned}
$$

where $\overline{\mathrm{T}}=0.3$ and $\left[\xi_{r 1}, \xi_{r 2}\right]^{T}=[1.2,0.7]^{T}$. Based on Theorem 11, the virtual stabilizing control laws and actual control laws are chosen as

$$
\begin{aligned}
s_{1,1}= & -0.8 z_{1,1} \omega_{1,1}-\frac{\widehat{\vartheta}_{1,1} z_{1,1} \oplus_{1,1}}{2 \times 0.25^{2}} \\
& -\widehat{\psi}_{1,1} \tanh \left(\frac{z_{1,1} \omega_{1,1}}{0.5}\right) \\
s_{2,1}= & -0.6 z_{2,1} \omega_{2,1}-\frac{\widehat{\vartheta}_{2,1} z_{2,1} \omega_{2,1}}{2 \times 0.1^{2}} \\
& -\widehat{\psi}_{2,1} \tanh \left(\frac{z_{2,1} \omega_{2,1}}{0.3}\right) \\
\iota_{1}= & -1.2 z_{1,2}-\frac{\widehat{\vartheta}_{1,2} z_{1,2}}{2 \times 0.5^{2}}-\widehat{\psi}_{1,2} \tanh \left(\frac{z_{1,2}}{0.75}\right) \\
\iota_{2}= & -1.5 z_{2,2}-\frac{\widehat{\vartheta}_{2,2} z_{2,2}}{2 \times 0.3^{2}}-\widehat{\psi}_{2,2} \tanh \left(\frac{z_{2,2}}{0.55}\right)
\end{aligned}
$$

with adaptation laws

$$
\begin{aligned}
& \dot{\widehat{\vartheta}}_{1,2}=\frac{z_{1,2}^{2}}{2 \times 0.5^{2}}-0.3 \widehat{\vartheta}_{1,2} \text {, } \\
& \dot{\hat{\psi}}_{1,2}=1.2\left[z_{1,2} \tanh \left(\frac{z_{1,2}}{0.75}\right)-0.3 \widehat{\psi}_{1,2}\right] \\
& \dot{\widehat{\vartheta}}_{1,1}=\frac{\left(z_{1,1} \omega_{1,1}\right)^{2}}{2 \times 0.25^{2}}-0.3 \widehat{\vartheta}_{1,1} \text {, } \\
& \dot{\hat{\psi}}_{1,1}=z_{1,1} \omega_{1,1} \tanh \left(\frac{z_{1,1} \omega_{1,1}}{0.5}\right)-0.3 \widehat{\psi}_{1,1} \\
& \dot{\widehat{\vartheta}}_{2,2}=1.5\left[\frac{z_{2,2}^{2}}{2 \times 0.3^{2}}-0.25 \widehat{\vartheta}_{2,2}\right] \text {, } \\
& \dot{\hat{\psi}}_{2,2}=2\left[z_{2,2} \tanh \left(\frac{z_{2,2}}{0.55}\right)-0.25 \widehat{\psi}_{2,2}\right] \\
& \dot{\hat{\vartheta}}_{2,1}=1.5\left[\frac{\left(z_{2,1} \omega_{2,1}\right)^{2}}{2 \times 0.1^{2}}-0.25 \widehat{\vartheta}_{2,1}\right] \text {, } \\
& \dot{\hat{\psi}}_{2,1}=1.2\left[z_{2,1} \omega_{2,1} \tanh \left(\frac{z_{2,1} \omega_{2,1}}{0.3}\right)-0.25 \widehat{\psi}_{2,1}\right]
\end{aligned}
$$

and performance functions

$$
\begin{aligned}
& \Omega_{1}(t)=-\Psi_{1}(t)=(1.1-0.07) e^{-t}+0.07 \\
& \Omega_{2}(t)=-\Psi_{2}(t)=(0.6-0.06) e^{-t}+0.06
\end{aligned}
$$

Choose the initial conditions as $\left[\xi_{1,1}(0), \xi_{1,2}(0)\right]^{T}=$ $[0.7,-0.7]^{T},\left[\xi_{2,1}(0), \xi_{2,2}(0)\right]^{T}=[0.3,-0.3]^{T}, \widehat{\vartheta}_{1,1}(0)=$ $\widehat{\vartheta}_{1,2}(0)=\widehat{\vartheta}_{2,1}(0)=\widehat{\vartheta}_{2,2}(0)=0$ and $\widehat{\psi}_{1,1}(0)=\widehat{\psi}_{1,2}(0)=$ $\widehat{\psi}_{2,1}(0)=\widehat{\psi}_{2,2}(0)=0$. The simulation results of Example 12 are shown in Figures 1-5.

Figure 1 shows that the system outputs $y_{1}$ and $y_{2}$ follow the desired trajectories $r_{1}$ and $r_{2}$ as closely as possible, and 

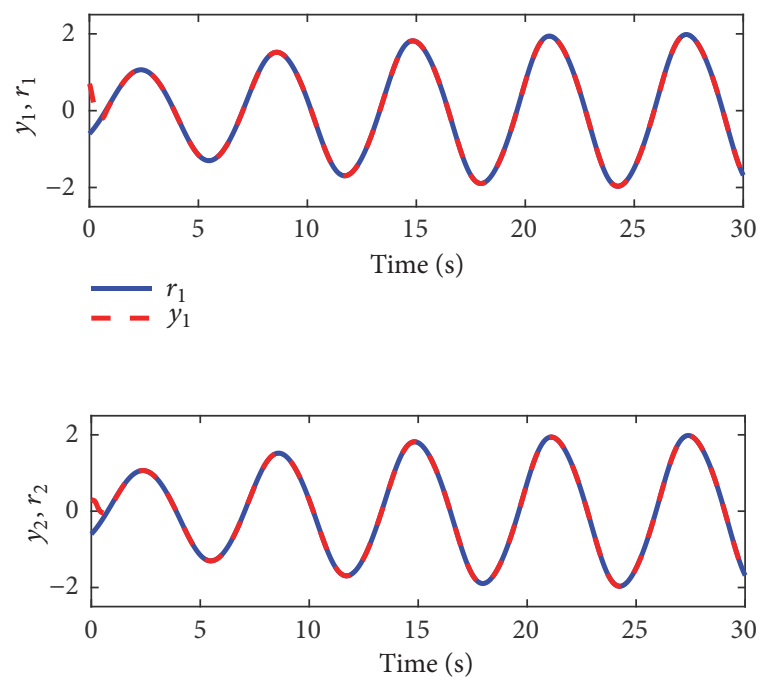

$r_{2}$
$-\quad y_{2}$

FIGURE 1: System outputs and reference signals.
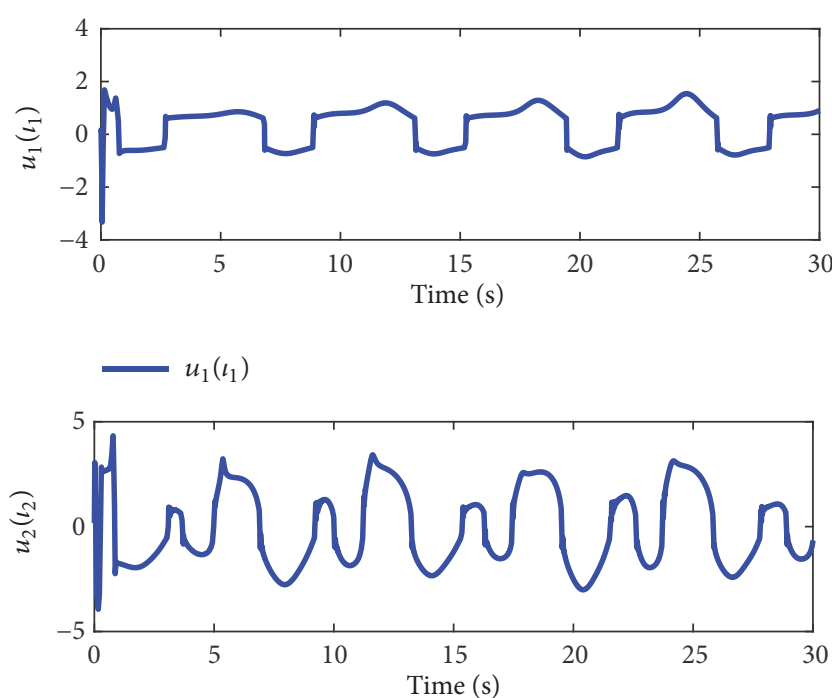

$-u_{2}\left(\iota_{2}\right)$

FIGURE 2: Curves of control inputs.

the control inputs $u_{1}\left(l_{1}\right)$ and $u_{2}\left(l_{2}\right)$ are shown in Figure 2. The prescribed performance bounds $\Omega_{1}, \Psi_{1}, \Omega_{2}, \Psi_{2}$ and the tracking errors $e_{1,1}, e_{2,1}$ are illustrated in Figures 3-4, from which we can see the output tracking errors achieve preselected transient and steady bounds. Finally, Figure 5 is given to explain the boundedness of adaptive parameters $\widehat{\vartheta}_{1,1}$, $\widehat{\psi}_{1,1}, \widehat{\vartheta}_{1,2}, \widehat{\psi}_{1,2}, \widehat{\vartheta}_{2,1}, \widehat{\psi}_{2,1}, \widehat{\vartheta}_{2,2}$, and $\widehat{\psi}_{2,2}$.

Example 13. Consider the following uncertain nonlinear systems

$$
\dot{\xi}_{1,1}=h_{1,1}\left(\bar{\xi}_{1,2}\right)+\theta_{1,1}\left(\bar{\xi}_{1,1}\right) \xi_{1,2}+D_{1,1}(\xi, t)
$$

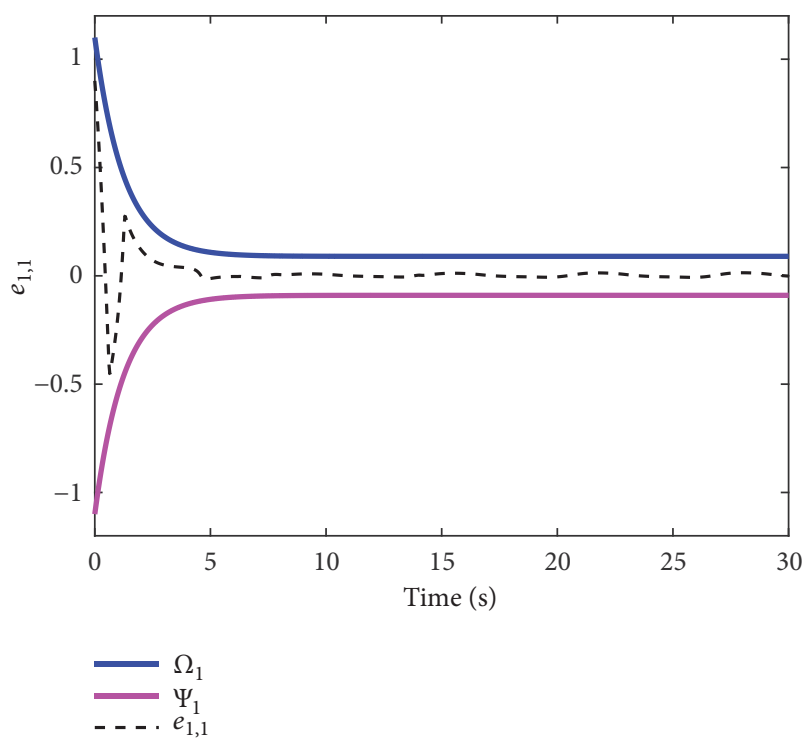

FIgURE 3: Curves of $e_{1,1}$ and performance bounds.

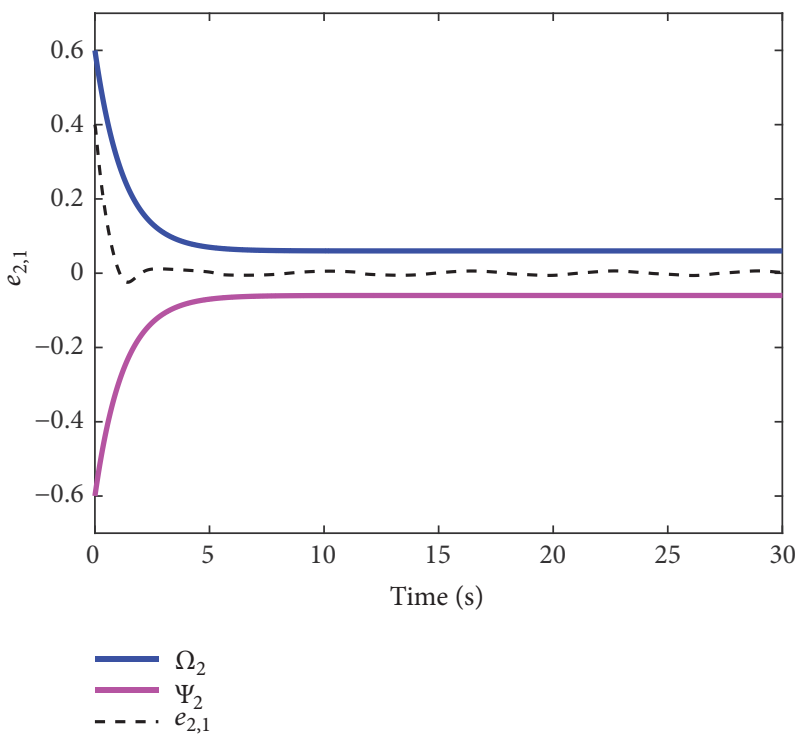

FIGURE 4: Curves of $e_{2,1}$ and performance bounds.

$$
\begin{aligned}
\dot{\xi}_{1,2} & =h_{1,2}\left(\bar{\xi}_{1,2}\right)+\theta_{1,2}\left(\bar{\xi}_{1,2}\right) u_{1}\left(\iota_{1}\right)+D_{1,2}(\xi, t) \\
\dot{\xi}_{2,1} & =h_{2,1}\left(\bar{\xi}_{2,2}\right)+\theta_{2,1}\left(\bar{\xi}_{2,1}\right) \xi_{2,2}+D_{2,1}(\xi, t) \\
\dot{\xi}_{2,2} & =h_{2,2}\left(\bar{\xi}_{2,2}\right)+\theta_{2,2}\left(\bar{\xi}_{2,2}\right) u_{2}\left(\iota_{2}\right)+D_{2,2}(\xi, t) \\
y_{1} & =\xi_{1,1} \\
y_{2} & =\xi_{2,1}
\end{aligned}
$$

where $h_{1,1}\left(\bar{\xi}_{1,2}\right)=\xi_{1,1}+\xi_{1,2}, h_{1,2}\left(\bar{\xi}_{1,2}\right)=\cos \left(\xi_{1,1}^{2} \xi_{1,2}^{2}\right) \xi_{1,2}+$ $\xi_{1,1} \xi_{1,2}^{2}, h_{2,1}\left(\bar{\xi}_{2,2}\right)=\xi_{1,1}+\xi_{1,2} \xi_{2,1} e^{\xi_{2,1}}, h_{2,2}\left(\bar{\xi}_{2,2}\right)=\xi_{2,1}+\xi_{1,2} \xi_{2,2}^{2}$ and $\theta_{2,1}\left(\bar{\xi}_{2,1}\right)=e^{\xi_{2,1}}, \theta_{1,1}\left(\bar{\xi}_{1,1}\right)=1.3+e^{\xi_{1,1}^{2}}, \theta_{1,2}\left(\bar{\xi}_{1,2}\right)=0.5+$ 

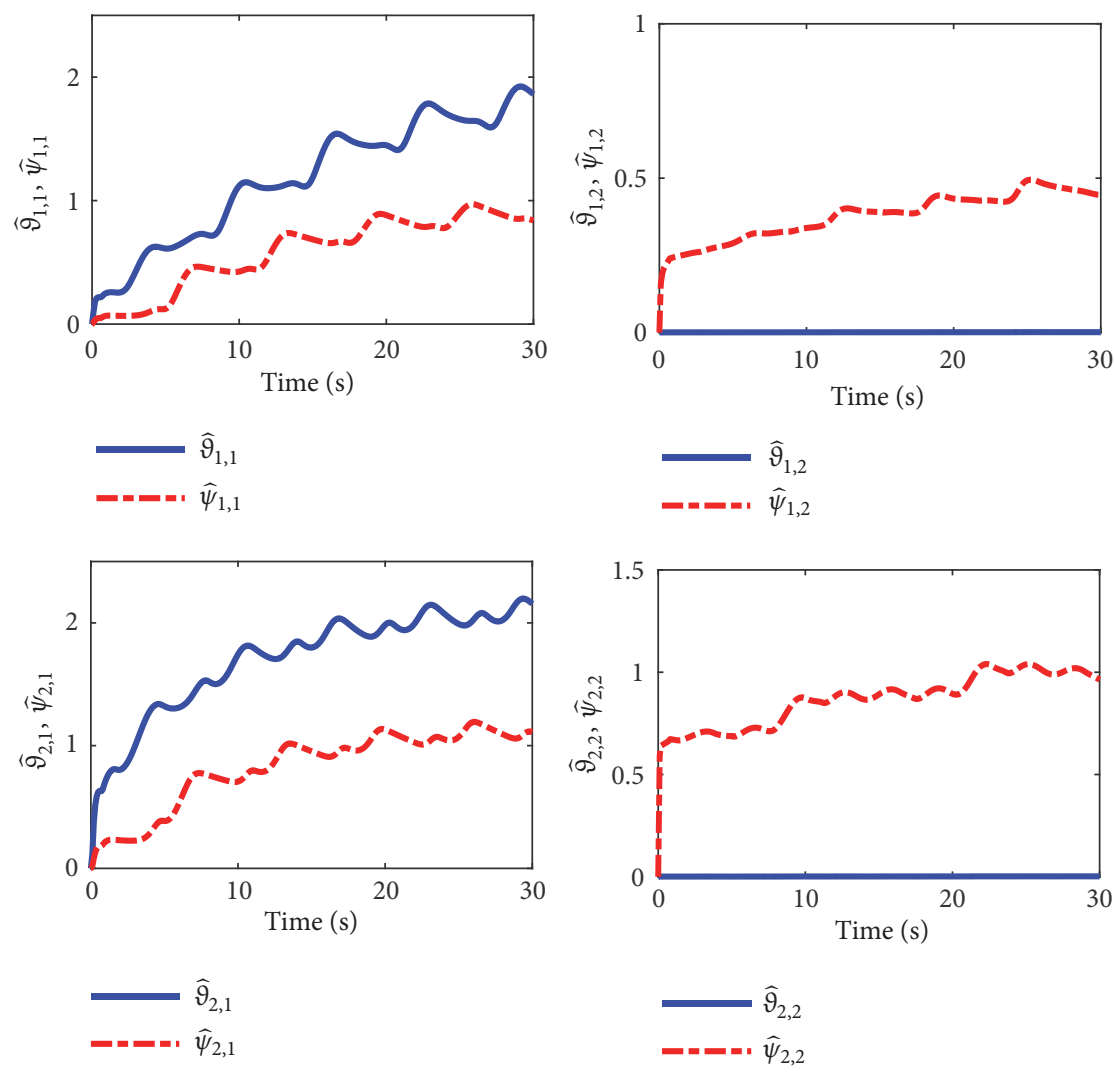

Figure 5: Curves of adaptive parameters.

$e^{\xi_{1,1} \xi_{1,2}}, \theta_{2,2}\left(\bar{\xi}_{2,2}\right)=1.2+e^{\xi_{1,1} \xi_{2,1} \xi_{2,2}} \cdot D_{1,1}=0.5 \cos (t) \sin (0.5 t)+$ $\xi_{1,1} \xi_{2,1} \xi_{2,2}^{2}, D_{1,2}=\xi_{1,2} \xi_{2,1}+0.3 \sin (t)^{2} \cos (t)+\xi_{1,1}^{2}, D_{2,1}=$ $0.5 \sin (t)+\xi_{1,2} \xi_{2,1}^{2} \xi_{2,2}$ and $D_{2,2}=\cos (0.2 t)(\sin (t))^{2}+\xi_{1,1} \xi_{1,2}+$ $\xi_{2,2}^{2} \cdot u_{1}\left(\iota_{1}\right)$ and $u_{2}\left(\iota_{2}\right)$ are described by

$$
\begin{aligned}
& u_{1}\left(\iota_{1}\right)=\operatorname{sat}\left(\iota_{1}\right)= \begin{cases}\operatorname{sign}\left(\iota_{1}\right) 4, & \iota_{1} \geq 4 \\
\iota_{1}, & \iota_{1}<4\end{cases} \\
& u_{2}\left(\iota_{2}\right)=\operatorname{sat}\left(\iota_{2}\right)= \begin{cases}\operatorname{sign}\left(\iota_{2}\right) 5.5, & \iota_{2} \geq 5.5 \\
\iota_{2}, & \iota_{2}<5.5\end{cases}
\end{aligned}
$$

Then, the virtual stabilizing control laws and actual control laws are constructed as

$$
\begin{aligned}
s_{1,1}= & -2 z_{1,1} \omega_{1,1}-\frac{\widehat{\vartheta}_{1,1} z_{1,1} \omega_{1,1}}{2 \times 0.5^{2}}-\widehat{\psi}_{1,1} \tanh \left(\frac{z_{1,1} \omega_{1,1}}{0.75}\right) \\
s_{2,1}= & -2.5 z_{2,1} \omega_{2,1}-\frac{\widehat{\vartheta}_{2,1} z_{2,1} \omega_{2,1}}{2 \times 0.1^{2}} \\
& -\widehat{\psi}_{2,1} \tanh \left(\frac{z_{2,1} \omega_{2,1}}{0.25}\right) \\
\iota_{1}= & -3 z_{1,2}-\frac{\widehat{\vartheta}_{1,2} z_{1,2}}{2 \times 0.25^{2}}-\widehat{\psi}_{1,2} \tanh \left(\frac{z_{1,2}}{0.75}\right) \\
\iota_{2}= & -4 z_{2,2}-\frac{\widehat{\vartheta}_{2,2} z_{2,2}}{2 \times 0.2^{2}}-\widehat{\psi}_{2,2} \tanh \left(\frac{z_{2,2}}{0.45}\right)
\end{aligned}
$$

with adaptive laws

$$
\begin{aligned}
& \dot{\hat{\psi}}_{1,2}=1.4\left[z_{1,2} \tanh \left(\frac{z_{1,2}}{0.75}\right)-0.4 \widehat{\psi}_{1,2}\right], \\
& \dot{\widehat{\vartheta}}_{1,2}=\frac{z_{1,2}^{2}}{2 \times 0.25^{2}}-0.4 \widehat{\vartheta}_{1,2} \\
& \dot{\widehat{\psi}}_{1,1}=z_{1,1} \omega_{1,1} \tanh \left(\frac{z_{1,1} \omega_{1,1}}{0.75}\right)-0.4 \widehat{\psi}_{1,1}, \\
& \dot{\widehat{\vartheta}}_{1,1}=1.3\left[\frac{\left(z_{1,1} \omega_{1,1}\right)^{2}}{2 \times 0.5^{2}}-0.4 \widehat{\vartheta}_{1,1}\right] \\
& \dot{\widehat{\psi}}_{2,2}=1.5\left[z_{2,2} \tanh \left(\frac{z_{2,2}}{0.45}\right)-0.35 \widehat{\psi}_{2,2}\right], \\
& \dot{\widehat{\vartheta}}_{2,2}=1.5\left[\frac{z_{2,2}^{2}}{2 \times 0.2^{2}}-0.35 \widehat{\vartheta}_{2,2}\right] \\
& \dot{\widehat{\psi}}_{2,1}=1.1\left[z_{2,1} \Phi_{2,1} \tanh \left(\frac{z_{2,1} \omega_{2,1}}{0.25}\right)-0.35 \widehat{\psi}_{2,1}\right], \\
& \dot{\vartheta}_{2,1}=2\left[\frac{\left(z_{2,1} \omega_{2,1}\right)^{2}}{2 \times 0.1^{2}}-0.35 \widehat{\vartheta}_{2,1}\right]
\end{aligned}
$$

and performance functions

$$
\Omega_{1}(t)=(0.5-0.08) e^{-t}+0.08
$$




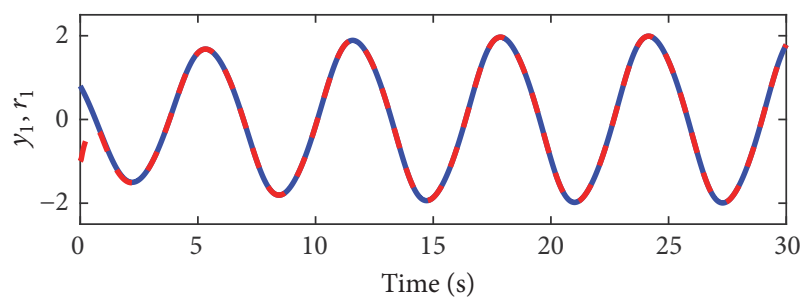

- $r_{1}$

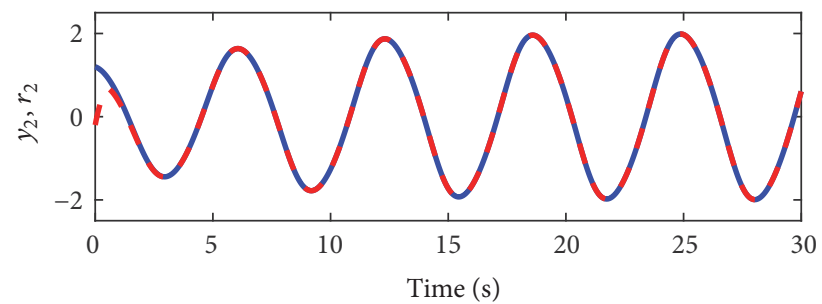

$r_{2}$
$-\quad y_{2}$

FIGURE 6: System outputs and reference signals.
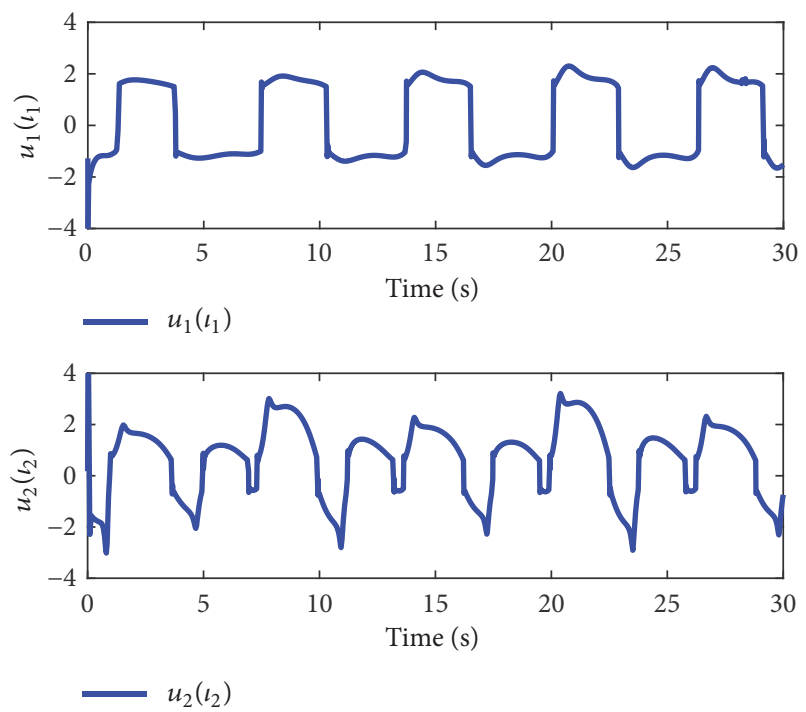

Figure 7: Curves of control inputs.

$$
\begin{aligned}
& \hbar_{1}(t)=(-2+0.08) e^{-t}-0.08 \\
& \Omega_{2}(t)=(1-0.09) e^{-2 t}+0.09 \\
& \hbar_{2}(t)=(-3+0.09) e^{-2 t}-0.09
\end{aligned}
$$

where the initial conditions $\left[\xi_{1,1}(0), \xi_{1,2}(0)\right]^{T}=[-0.9,0.9]^{T}$, $\left[\xi_{2,1}(0), \xi_{2,2}(0)\right]^{T}=[-0.2,0.2]^{T}, \widehat{\vartheta}_{1,1}(0)=\widehat{\vartheta}_{1,2}(0)=\widehat{\vartheta}_{2,1}(0)=$ $\widehat{\vartheta}_{2,2}(0)=0$, and $\widehat{\psi}_{1,1}(0)=\widehat{\psi}_{1,2}(0)=\widehat{\psi}_{2,1}(0)=\widehat{\psi}_{2,2}(0)=0$. The simulation results of Example 13 are shown in Figures 6-9.

Figure 6 shows that the system outputs track the desired trajectories, perfectly, and Figure 7 reveals that the system inputs are bounded. From Figures 8-9, the tracking errors
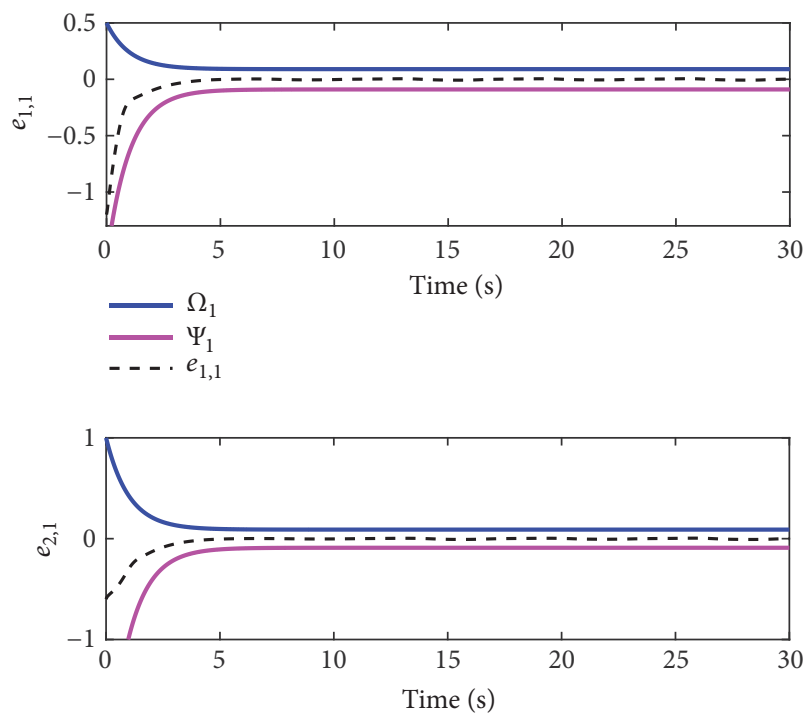

$-\Omega_{2}$
$-\ldots e_{2,1}$

FIGURE 8: Tracking errors and performance bounds.
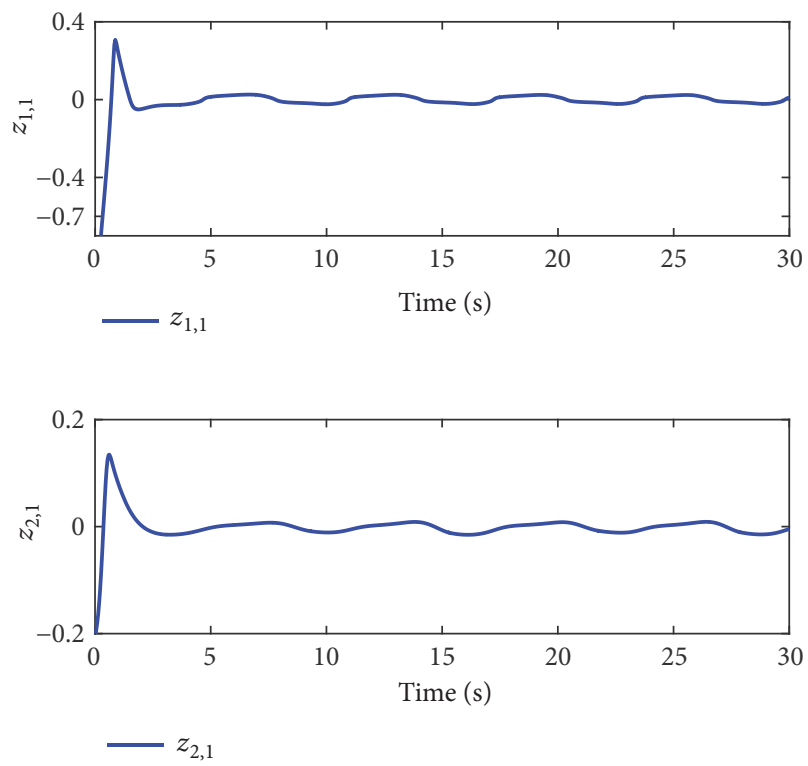

FIGURE 9: Curves of transformed errors $z_{1,1}$ and $z_{2,1}$.

$e_{1,1}, e_{2,1}$ satisfy the asymmetry prescribed bounds and the transformed errors $z_{1,1}, z_{2,1}$ are bounded, from which we can see that a fairly good tracking performance is achieved.

\section{Conclusion}

This paper proposed a novel adaptive neural PPC scheme for large classes of nonlinear nonstrict-feedback systems with prescribed performance under the effect of input saturation. Compared with previously published methods [27-43], the restrictive assumption that the upper and lower bounds of 
control gain functions must be positive constants or coefficients is relieved under the proposed method. The innovative error transformation proposed in this paper also overcomes the nondifferentiable obstacle and complex deductions corresponding to traditional PPC schemes. A novel auxiliary system with a bounded compensation term was also proposed to relax the strictly bounded assumption of the dynamic system. The stability of the closed-loop system was proven under Lyapunov and invariant set theories. Tracking errors were made arbitrarily small by appropriately selecting the design parameters. Finally, the simulation results show that the proposed control laws do achieve the desired performance. In the future, we will address control problems for uncertain nonlinear systems with an unbounded or nondifferentiable time derivative of disturbance; this will allow us to utilize the PPC approach combined with set-invariance mechanisms.

\section{Appendix}

Proof of Lemma 9. From $\Psi_{j}(0)<e_{j, 1}(0)<\Omega_{j}(0)$ and (8), we have $-1<\lambda_{\mathrm{j}, 1}(0)<1$.

Case $1\left(0 \leq v_{j, 1}(t) \leq \bar{v}_{j, 1}\right)$. According to (7) and $0 \leq v_{j, 1}(t) \leq$ $\bar{v}_{j, 1}$, we can obtain (i) $\lambda_{j, 1}(t) \geq 0,1-\lambda_{j, 1}^{2}(t)>0$ for $\forall t \geq 0$; (ii) $\lambda_{j, 1}(t) \leq 0,1-\lambda_{j, 1}^{2}(t)<0$ for $\forall t \geq 0$. Clearly, there is a contradiction between (ii) and $-1<\lambda_{j, 1}(0)<1$.

Case $2\left(-\bar{v}_{j, 1} \leq v_{j, 1}(t)<0\right)$. According to $(7)$ and $-\bar{\nu}_{j, 1} \leq$ $v_{j, 1}(t)<0$, we can obtain (i) $\lambda_{j, 1}(t) \leq 0,1-\lambda_{j, 1}^{2}(t)>0$ for $\forall t \geq 0$; (ii) $\lambda_{j, 1}(t) \geq 0,1-\lambda_{j, 1}^{2}(t)<0$ for $\forall t \geq 0$. Clearly, there is a contradiction between (ii) and $-1<\lambda_{j, 1}(0)<1$.

Thus, if $\Psi_{j}(0)<e_{j, 1}(0)<\Omega_{j}(0)$ and $\left|\nu_{j, 1}(\mathrm{t})\right| \leq \bar{\nu}_{j, 1}$, we will obtain $-1<\lambda_{j, 1}(\mathrm{t})<1$ for $\forall t \geq 0$. From (8), we can have $\Psi_{j}(0)<e_{j, 1}(0)<\Omega_{j}(0)$ for $\forall t \geq 0$. This completes the proof.

Proof of Lemma 10. Observing $e_{j, 1}=\xi_{j, 1}-r_{j}$, we have $\xi_{j, 1}=$ $e_{j, 1}+r_{j}$, so that the function $\theta_{j, 1}\left(\xi_{j, 1}\right)$ can be expressed by

$$
\theta_{j, 1}\left(\xi_{j, 1}\right)=\Phi_{j, 1}\left(e_{j, 1}, r_{j}\right)
$$

with $\Phi_{j, 1}(\cdot)$ being a continuous function of $e_{j, 1}$ and $r_{j}$. Note that $\Pi_{j, 1} \times \Pi_{j 0}$ is a compact set since $\Pi_{j, 1}$ and $\Pi_{j 0}$ are compact sets. Furthermore, it can be seen from (A.1) that all the variables of $\Phi_{j, 1}\left(e_{j, 1}, r_{j}\right)$ are included in $\Pi_{j, 1} \times \Pi_{j 0}$. Thus, we have

$$
0<\underline{\theta}_{j, 1} \leq \theta_{j, 1}\left(\xi_{j, 1}\right) \leq \bar{\theta}_{j, 1}, \quad \xi_{j, 1} \in \Pi_{j, 1} \times \Pi_{j 0}
$$

This completes the proof.

\section{Data Availability}

No data were used to support this study.

\section{Conflicts of Interest}

The authors declare that there are no conflicts of interest regarding the publication of this paper.

\section{Acknowledgments}

This work is supported by the National Social Science Foundation of China (Grant no. 14GJ003-173) and the National Natural Science Foundation of China (Grant no. 71601183).

\section{References}

[1] X. Zhao, H. Yang, W. Xia, and X. Wang, "Adaptive fuzzy hierarchical sliding-mode control for a class of MIMO nonlinear time-delay systems with input saturation," IEEE Transactions on Fuzzy Systems, vol. 25, no. 5, pp. 1062-1077, 2017.

[2] Y. Li, S. Tong, L. Liu, and G. Feng, "Adaptive output-feedback control design with prescribed performance for switched nonlinear systems," Automatica, vol. 80, pp. 225-231, 2017.

[3] X. F. Meng, Y. Wang, and M. L. Lv, "Adaptive NN control for multisteering plane aircraft with dead zone or backlash input nonlinearity," Mathematical Problems in Engineering, vol. 2017, Article ID 4684303, 8 pages, 2017.

[4] Y.-J. Liu, J. Li, S.-C. Tong, and C. L. P. Chen, "Neural network control-based adaptive learning design for nonlinear systems with full-state constraints," IEEE Transactions on Neural Networks and Learning Systems, vol. 27, no. 7, pp. 1562-1571, 2016.

[5] S. Baldi, C. D. Korkas, M. Lv, and E. B. Kosmatopoulos, "Automating occupant-building interaction via smart zoning of thermostatic loads: A switched self-tuning approach," Applied Energy, vol. 231, pp. 1246-1258, 2018.

[6] M. R. Rosa, S. Baldi, X. Wang, M. Lv, and W. Yu, "Adaptive hierarchical formation control for uncertain Euler-Lagrange systems using distributed inverse dynamics," European Journal of Control, vol. 48, pp. 52-65, 2019.

[7] L. Zhang, S. Sui, Y. Li, and S. Tong, "Adaptive fuzzy output feedback tracking control with prescribed performance for chemical reactor of MIMO nonlinear systems," Nonlinear Dynamics, vol. 80, no. 1-2, pp. 945-957, 2015.

[8] J. Zhang, B. Xiao, M. Lv, and Q. Zhang, "Design and flightstability analysis of a closed fixed-wing unmanned aerial vehicle formation controller," Proceedings of the Institution of Mechanical Engineers, Part I: Journal of Systems and Control Engineering, 2018.

[9] F. Wang, B. Chen, X. Liu, and C. Lin, "Finite-time adaptive fuzzy tracking control design for nonlinear systems," IEEE Transactions on Fuzzy Systems, vol. 26, no. 3, pp. 1207-1216, 2018.

[10] N. Wang, Y. Wang, X. Wang, and Z. Liu, "FSE-RBFNNs-based robust adaptive DSC design for a larger class of nonlinear strictfeedback systems," IEEE Access, vol. 7, pp. 63361-63372, 2019.

[11] M.-1. Lv, X.-x. Sun, S.-g. Liu, and D. Wang, "Adaptive tracking control for non-affine nonlinear systems with non-affine function possibly being discontinuous," International Journal of Systems Science, vol. 48, no. 5, pp. 1115-1122, 2017.

[12] M. Krstić, I. Kanellakopoulos, and P. V. Kokotović, "Adaptive nonlinear control without overparametrization," Systems \& Control Letters, vol. 19, no. 3, pp. 177-185, 1992.

[13] D. Seto, A. Annaswamy, and J. Baillieul, "Adaptive control of nonlinear systems with a triangular structure," IEEE Transactions on Automatic Control, vol. 39, no. 7, pp. 1411-1428.

[14] M. L. Lv, X. X. Sun, and S. G. Liu, "An adaptive dynamic surface controller for ultralow altitude airdrop flight path angle with actuator input nonlinearity," Mathematical Problems in Engineering, vol. 2016, Article ID 4753241, 9 pages, 2016. 
[15] B. Niu and L. Li, "Adaptive backstepping-based neural tracking control for MIMO nonlinear switched systems subject to input delays," IEEE Transactions on Neural Networks and Learning Systems, vol. 29, no. 6, pp. 2638-2644, 2018.

[16] S. S. Pavlichkov, S. N. Dashkovskiy, and C. K. Pang, "Uniform stabilization of nonlinear systems with arbitrary switchings and dynamic uncertainties," IEEE Transactions on Automatic Control, vol. 62, no. 5, pp. 2207-2222, 2017.

[17] M. Lv, S. Baldi, and Z. Liu, "The non-smoothness problem in disturbance observer design: a set-invariance-based adaptive fuzzy control method," IEEE Transactions on Fuzzy Systems, vol. 27, no. 3, pp. 598-604, 2019.

[18] J. Zhang, J. Yan, M. Lv, X. Kong, and P. Zhang, "UAV formation flight cooperative tracking controller design," in Proceedings of the 15th International Conference on Control, Automation, Robotics and Vision, ICARCV 2018, pp. 856-861, Singapore, November 2018.

[19] B. Chen, K. F. Liu, X. P. Liu, P. Shi, and C. Lin, "Approximationbased adaptive neural control design for a class of nonlinear systems," IEEE Transactions on Cybernetics, vol. 25, no. 1, pp. 111-123, 2014.

[20] H. Wang, X. Liu, K. Liu, and H. R. Karimi, "Approximationbased adaptive fuzzy tracking control for a class of nonstrictfeedback stochastic nonlinear time-delay systems," IEEE Transactions on Fuzzy Systems, vol. 23, no. 5, pp. 1746-1760, 2015.

[21] M. Lv, Y. Wang, S. Baldi et al., "Adaptive neural control for pure feedback nonlinear systems with uncertain actuator nonlinearity," in Proceedings of the International Conference on Neural Information Processing, vol. 6 of Lecture Notes in Computer Science, pp. 201-211, Springer International Publishing, Guangzhou, China, 2017.

[22] M. Lv, Y. Wang, S. Baldi, Z. Liu, and Z. Wang, "A DSC method for strict-feedback nonlinear systems with possibly unbounded control gain functions," Neurocomputing, vol. 275, pp. 13831392, 2018.

[23] Q. Li, R. Yang, and Z. Liu, "Adaptive tracking control for a class of nonlinear non-strict-feedback systems," Nonlinear Dynamics, vol. 88, no. 3, pp. 1537-1550, 2017.

[24] C. P. Bechlioulis and G. A. Rovithakis, "Robust adaptive control of feedback linearizable MIMO nonlinear systems with prescribed performance," IEEE Transactions on Automatic Control, vol. 53, no. 9, pp. 2090-2099, 2008.

[25] C. P. Bechlioulis and G. A. Rovithakis, "Adaptive control with guaranteed transient and steady state tracking error bounds for strict feedback systems," Automatica, vol. 45, no. 2, pp. 532-538, 2009.

[26] S. I. Han and J. M. Lee, "Partial tracking error constrained fuzzy dynamic surface control for a strict feedback nonlinear dynamic system," IEEE Transactions on Fuzzy Systems, vol. 22, no. 5, pp. 1049-1061, 2014.

[27] J. Dhruv, M. Lv, and S. Baldi, "Hybrid adaptive chassis control for vehicle lateral stability in the presence of uncertainty," in Proceedings of the 26th Mediterranean Conference on Control and Automation, MED 2018, pp. 1-6, Zadar, Croatia, June 2018.

[28] S. I. Han and J. M. Lee, "Fuzzy echo state neural networks and funnel dynamic surface control for prescribed performance of a nonlinear dynamic system," IEEE Transactions on Industrial Electronics, vol. 61, no. 2, pp. 1099-1112, 2014.

[29] C. P. Bechlioulis and G. A. Rovithakis, "A priori guaranteed evolution within the neural network approximation set and robustness expansion via prescribed performance control,"
IEEE Transactions on Neural Networks and Learning Systems, vol. 23, no. 4, pp. 669-675, 2012.

[30] J. M. Lee and S. I. Han, "Improved prescribed performance constraint control for a strict feedback non-linear dynamic system," IET Control Theory \& Applications, vol. 7, no. 14, pp. 1818-1827, 2013.

[31] S. J. Yoo, "Fault-tolerant control of strict-feedback non-linear time-delay systems with prescribed performance," IET Control Theory \& Applications, vol. 7, no. 11, pp. 1553-1561, 2013.

[32] S. Tong, S. Sui, and Y. Li, "Fuzzy adaptive output feedback control of MIMO nonlinear systems with partial tracking errors constrained," IEEE Transactions on Fuzzy Systems, vol. 23, no. 4, pp. 729-742, 2014.

[33] C. Hua, L. Zhang, and X. Guan, "Decentralized output feedback adaptive NN tracking control for time-delay stochastic nonlinear systems with prescribed performance," IEEE Transactions on Neural Networks and Learning Systems, vol. 26, no. 11, pp. 27492759, 2015.

[34] B. S. Kim and S. J. Yoo, "Approximation-based adaptive tracking control of nonlinear pure-feedback systems with time-varying output constraints," International Journal of Control, Automation, and Systems, vol. 13, no. 2, pp. 257-265, 2015.

[35] S. Han and J. Lee, "Prescribed performance fuzzy backstepping control for nonlinear large-scale systems," International Journal of Control, Automation, and Systems, vol. 13, no. 6, pp. 15081520, 2015.

[36] M. Chen, G. Tao, and B. Jiang, "Dynamic surface control using neural networks for a class of uncertain nonlinear systems with input saturation," IEEE Transactions on Neural Networks and Learning Systems, vol. 26, no. 9, pp. 2086-2097, 2014.

[37] N. Wang, Y. Wang, and M. Lv, "Fuzzy adaptive DSC design for an extended class of MIMO pure-feedback non-affine nonlinear systems in the presence of input constraints," Mathematical Problems in Engineering, vol. 2019, Article ID 4360643, 14 pages, 2019.

[38] H. Jing-Lin, S. Xiu-Xia, L. Ri, D. Xiong-Feng, and L. MaoLong, "UAV real-time route planning based on multi-optimized RRT algorithm," in Proceedings of the 29th Chinese Control and Decision Conference, CCDC 2017, pp. 837-842, IEEE, Chongqing, China, May 2017.

[39] M. Lv, W. Yu, and S. Baldi, "The set-invariance paradigm in fuzzy adaptive DSC design of large-scale nonlinear inputconstrained systems," IEEE Transactions on Systems, Man, and Cybernetics: Systems, pp. 1-11, 2019.

[40] Q. Zhou, H. Li, C. Wu, L. Wang, and C. K. Ahn, "Adaptive fuzzy control of nonlinear systems with unmodeled dynamics and input saturation using small-gain approach," IEEE Transactions on Systems, Man, and Cybernetics: Systems, vol. 47, pp. 19791989, 2017.

[41] Q. Zhou, L. Wang, C. Wu, H. Li, and H. Du, "Adaptive fuzzy control for nonstrict-feedback systems with input saturation and output constraint," IEEE Transactions on Systems, Man, and Cybernetics: Systems, vol. 47, pp. 1-12, 2017.

[42] M. Lv, X. Sun, and D. Wang, "An adaptive DSC for ultra-low altitude airdrop path tracking with actuator input dead zone," in Proceedings of the 2016 35th Chinese Control Conference (CCC), pp. 404-409, IEEE, Chengdu, China, July 2016.

[43] H. Li, L. Bai, Q. Zhou, R. Lu, and L. Wang, "Adaptive fuzzy control of stochastic nonstrict-feedback nonlinear systems with input saturation," IEEE Transactions on Systems, Man, and Cybernetics: Systems, vol. 47, no. 8, pp. 2185-2197, 2017. 


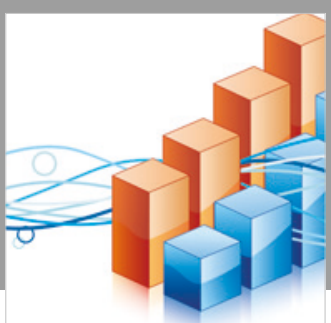

Advances in

Operations Research

\section{-n-m}
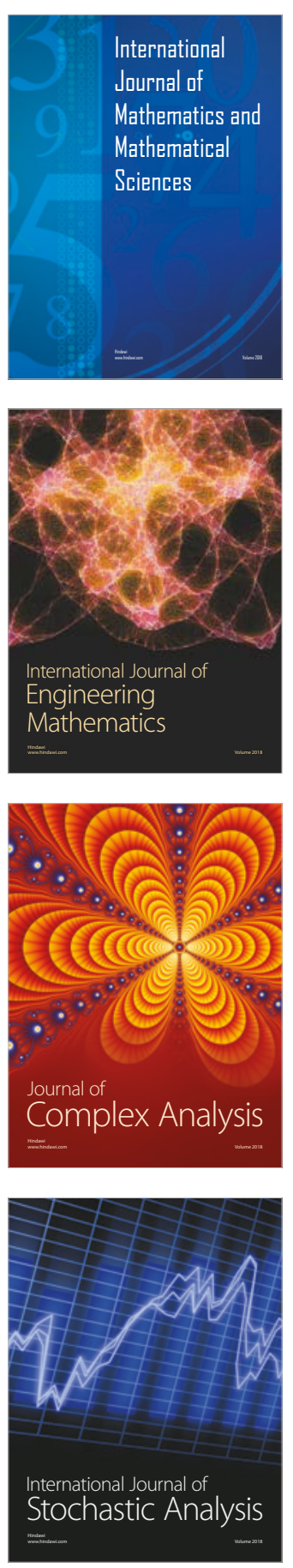
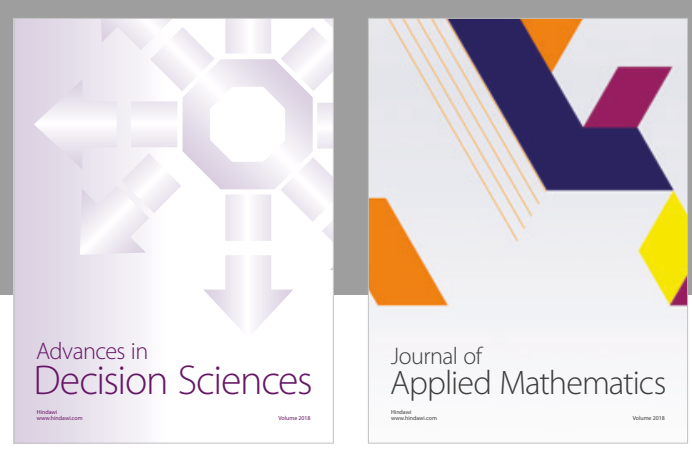

Journal of

Applied Mathematics
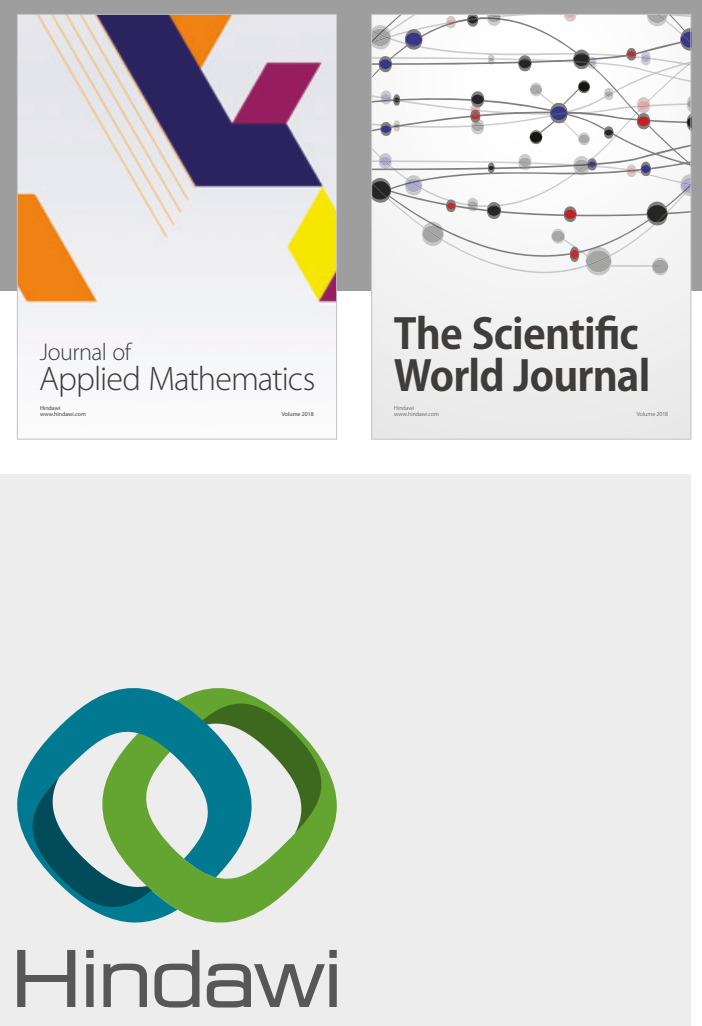

Submit your manuscripts at

www.hindawi.com

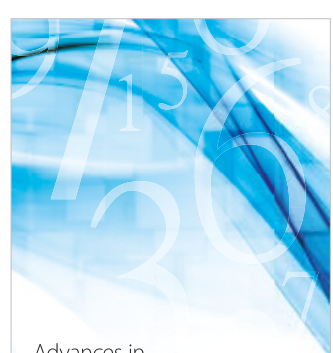

Advances in
Numerical Analysis
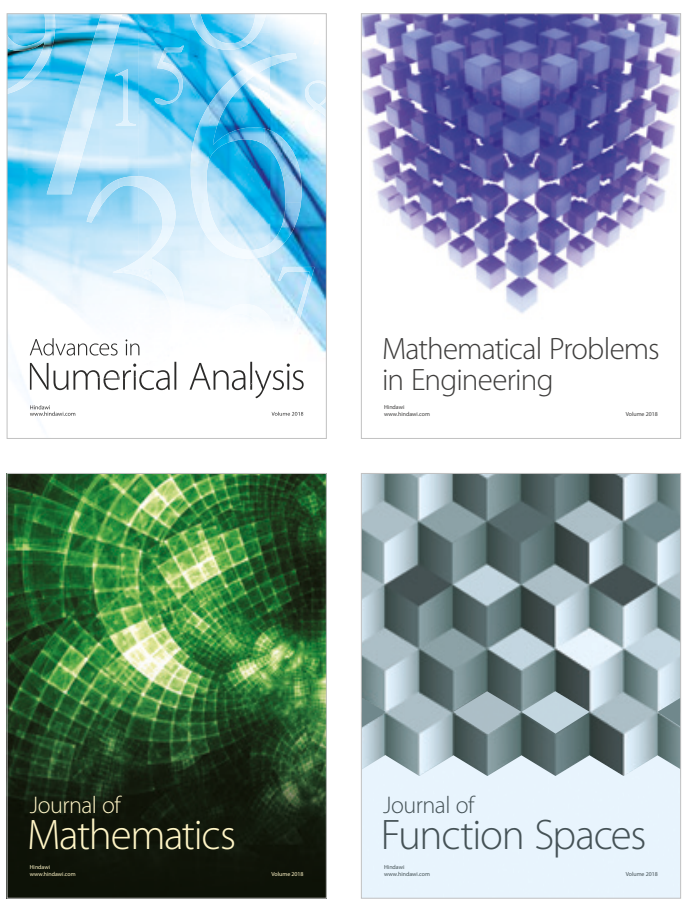

Mathematical Problems in Engineering

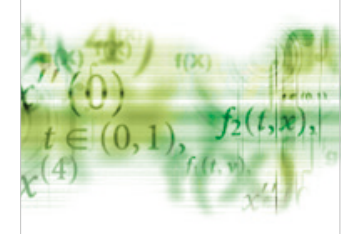

International Journal of

Differential Equations

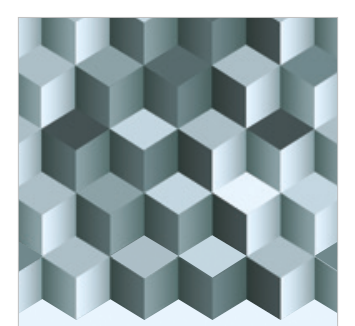

Journal of

Function Spaces

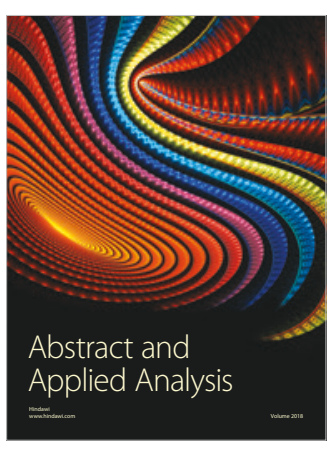

The Scientific

World Journal

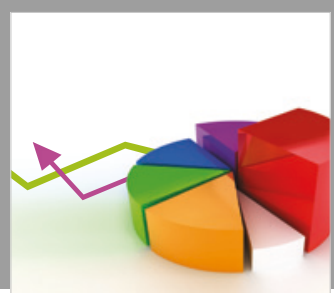

Journal of

Probability and Statistics
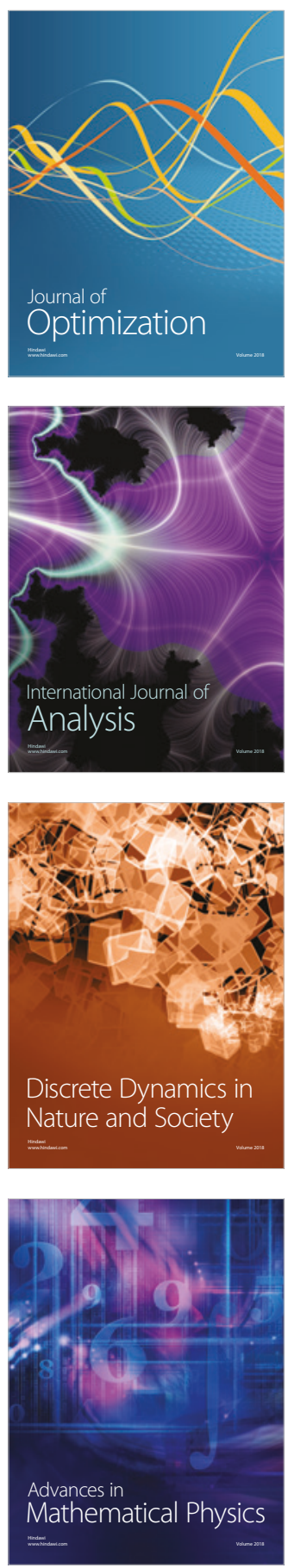